Check for updates

Cite this: Phys. Chem. Chem. Phys., 2018, 20, 6708

Received 23rd October 2017, Accepted 21st December 2017

DOI: $10.1039 / \mathrm{c} 7 \mathrm{cp} 07171 \mathrm{j}$

rsc.li/pccp

\section{On the photocatalytic cycle of water splitting with small manganese oxides and the roles of water clusters as direct sources of oxygen molecules $\dagger$}

\author{
Kentaro Yamamoto (D) and Kazuo Takatsuka (D) *
}

\begin{abstract}
We theoretically studied the chemical principles behind the photodynamics of water splitting: $2 \mathrm{H}_{2} \mathrm{O}+$ $4 h \nu+\mathrm{M} \rightarrow 4 \mathrm{H}^{+}+4 \mathrm{e}^{-}+\mathrm{O}_{2}+\mathrm{M}$. To comprehend this simple looking but very complicated reaction, the mechanisms of at least three crucial phenomena, among others, need to be clarified, each of which is supposed to constitute the foundation of chemistry: (i) charge separation $\left(4 \mathrm{H}^{+}+4 \mathrm{e}^{-}\right)$, (ii) the catalytic cycle for essentially the same reactions to be repeated by each of four photon absorptions with a catalyst M, and (iii) the generation of oxygen molecules of spin triplet. We have previously clarified the photodynamical mechanism of charge separation, which we refer to as coupled proton electronwavepacket transfer (CPEWT), based on the theory of nonadiabatic electron wavepacket dynamics [K. Yamamoto and K. Takatsuka, ChemPhysChem, 2017, 18, 537]. CPEWT gives an idea of how charge separation can be materialized at each single photon absorption. Yet, this mechanism alone cannot address the above crucial items such as (ii) the catalytic cycle and (iii) $\mathrm{O}_{2}$ formation. In the studies of these fundamental processes, we constructed a possible minimal chemical system and perform semiquantitative quantum chemical analyses, with which to attain insights about the possible mechanisms of photochemical water splitting. The present study has been inspired by the idea underlying the so-called Kok cycle, although we do not aim to simulate photosystem II in biological systems in nature. For instance, we assume here that a catalyst $M$ (actually simple manganese oxides in this particular study) is pumped up to its excited states leading to charge separation by four-time photon absorption, each excitation of which triggers individual series of chemical reactions including the reorganization of the hydrogen-bonding network (cluster) of water molecules surrounding the photocatalytic center. It is shown that in the successive processes of restructuring of the relevant water cluster, the $\mathrm{O}=\mathrm{O}$ bond is formed and consequently an oxygen molecule of spin triplet can be isolated within a range of a given photon energy of about $3.0 \mathrm{eV}$.
\end{abstract}

\section{Introduction}

Photoinduced water oxidation, summarized as:

$$
2 \mathrm{H}_{2} \mathrm{O}+4 h \nu+\mathrm{M} \rightarrow 4 \mathrm{H}^{+}+4 \mathrm{e}^{-}+\mathrm{O}_{2}+\mathrm{M},
$$

is a very fundamental process not only in biological systems but in the technology of water splitting. There are two ways for photon energy to be utilized in this system: (i) in one class, photoabsorption is realized elsewhere in the antenna subsystems and the photon energy is used to "pump up" electrons created in eqn (1), suggesting that the water splitting reactions proceed whilst keeping the catalytic center $\mathbf{M}$ in its ground state. This mechanism is widely believed to dominate

Fukui Institute for Fundamental Chemistry, Kyoto University, Sakyou-ku, Kyoto 606-8103, Japan.E-mail: kyamamoto@fukui.kyoto-u.ac.jp, kaztak@fukui.kyoto-u.ac.jp

$\dagger$ Electronic supplementary information (ESI) available. See DOI: 10.1039/c7cp07171j photosystem II (PSII) in plants and cyanobacteria. ${ }^{1,2}$ (ii) In the other class of reaction mechanism, the catalyst $\mathbf{M}$ (or a subsystem containing $\mathbf{M}$ ) is directly photoexcited, and is more explicitly depicted as:

$$
2 \mathrm{H}_{2} \mathrm{O}+4 h \nu+\mathrm{M} \rightarrow 2 \mathrm{H}_{2} \mathrm{O}+\mathrm{M}^{*} \rightarrow 4 \mathrm{H}^{+}+4 \mathrm{e}^{-}+\mathrm{O}_{2}+\mathrm{M}
$$

where $\mathbf{M}^{*}$ is the appropriate electronically excited state of $\mathbf{M}$. This direct excitation mechanism tends to be adopted in the study of artificial water splitting. The main aim of this work is not the simulation or direct analysis of the photosynthesis system but the study of the reaction mechanism of eqn (2). In this reaction, protons and electrons are created by charge separation due to the photoexcited catalytic center $\mathbf{M}^{*}$. Looking simple, this reaction involves crucial mysteries, which chemists should resolve in order to reveal the fundamental chemical principles. Among others, the following three things are critically 
important from a chemical point of view: (i) the mechanism of the charge separation $4 \mathrm{H}^{+}+4 \mathrm{e}^{-}$, (ii) the explicit scheme and functions of the catalytic cycle with $\mathrm{M}$ for the four-time photon process to continue, and (iii) the chemical mechanism to yield triplet oxygen molecules, and its where and how. Since the mechanism of photochemical charge separation in moieties of biological and/ or organic molecules has been clarified before, ${ }^{3-5}$ here we focus on the second and third questions: theoretical construction of the photochemical catalytic cycle with small $\mathrm{Mn}$ oxides (not $\mathrm{Mn}_{4} \mathrm{CaO}_{5}$ in PSII) and elucidation of the mechanism of spin-triplet $\mathrm{O}_{2}$ generation from the cycle.

For this goal we tried to construct a basic model of the photocatalytic cycle that can realize the reaction of eqn (2) after the cycle of four photon absorption is completed. This system is composed of (a) Mn oxide $\left(\mathrm{MnCaO}_{4} \mathrm{H}_{5}\right)$ as a photocatalytic center, (b) acceptors of protons and electrons after charge separation, (c) a hydrogen-bonding network (cluster) of water molecules surrounding and partially attached to the catalytic center, and (d) proton and electron buffering subsystems (molecules) to regulate the relevant oxidation-reduction reactions in the water cluster. We show in terms of this system that each photon absorption by the catalyst triggers individual series of chemical reactions including reorganization of the hydrogen-bonding network of water molecules and $\mathrm{O}-\mathrm{O}$ bond formation, as a peroxide in it can eventually lead to generation of spin-triplet $\mathrm{O}_{2}$. Therefore oxygen molecules are extracted out of abundant bulk water, which is stably and continuously supplied from the surroundings.

Our former studies related to water splitting have been mainly aimed at identification of the chemical dynamics of charge separation. Indeed, we have proposed a quantum mechanical mechanism of charge separation catalyzed by $\mathrm{Mn}$ oxides through nonadiabatic dynamics calculations in simple systems. $^{3-5}$ In these studies we have elucidated (i) the (quantum) dynamical mechanism(s) of charge separation to create protons and electrons, and (ii) the conditions that the proton-electron acceptor(s) should satisfy to enable the reaction to take place. For instance, the role of the Rydberg-like diffused vacant states on the nitrogen atoms involved in the amino acid residues which act as electron acceptors, has been clarified. More precisely, the proton involved in the relay-transfer is not bare but covered with as many as around 0.5 electrons as in ground-state proton transfer. ${ }^{6}$ The residual $\sim 0.5$ electrons are transferred to the Rydberg-like states of the EA through different pathways of the proton relay-transfer. As a result, approximately \pm 0.5 charge separation is induced on the different acceptor molecules. We refer to the above mechanism as coupled proton electron-wavepacket transfer (CPEWT) to stress that we focus on dynamics that can be properly characterized by the theoretical framework of nonadiabatic electron-wavepacket dynamics. Also, particularly relevant to the present work is the charge transfer in the largest extended system with Y-shaped proton-electron acceptors, ${ }^{5}$ which is schematically expressed as:

$$
\begin{array}{r}
\Omega-\mathrm{OH}_{2} \cdots[\mathrm{EA}-\mathrm{H}]^{+} \cdots[\mathrm{PA}]^{-} \stackrel{h \nu}{\longrightarrow} \\
\Omega^{\bullet}-\mathrm{OH} \cdots[\mathrm{H}-\mathrm{EA}]^{\bullet *} \cdots \mathrm{H}-\mathrm{PA}
\end{array}
$$

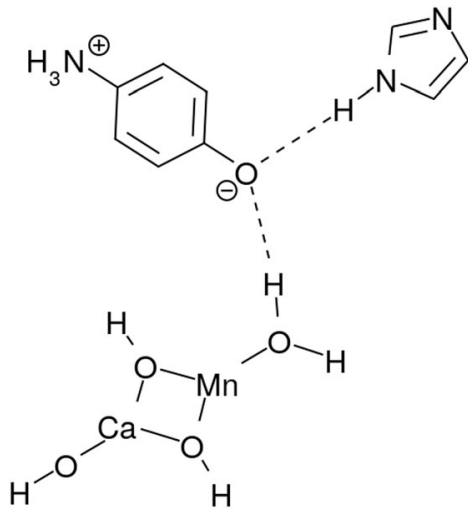

Fig. 1 A charge separation system studied in ref. 5 as a study of $Y$-shaped electron-proton acceptors.

in which $\Omega=\mathrm{MnOH}$ or $\mathrm{MnCa}(\mathrm{OH})_{3}$ (electron-proton donor), $\mathrm{EA}^{+}=4$-hydroxyanilinium cation (electron acceptor), and $\mathrm{PA}^{-}=$ imidazolate anion (proton acceptor) (see Fig. 1 for the stable intermediate).

Incidentally the definition of "proton-coupled electron transfer" (PCET) by Hammes-Schiffer indicates a general phenomena including any type of mechanism in which proton and electron transfers somehow couple with each other. ${ }^{7,8}$ The dynamical mechanism illustrated in eqn (3) is also included within PCET by definition. However, PCET covers too broad a range to differentiate the precise phenomena and mechanisms. PCET has been widely studied, ${ }^{9-15}$ but among these studies, those of Domcke and coworkers ${ }^{16-32}$ are particularly relevant to the present work. They have extensively investigated static properties along the excited-state potential curves to consider mechanisms related to excited-state proton and/or electron transfer. Their studies have also covered artificial photochemical water-splitting. ${ }^{23-32}$ Water-splitting in this context is recognized as a photoinduced homolytic dissociation to obtain $\mathrm{H}^{\bullet}$ and $\mathrm{OH}^{\bullet}$. This photochemical reaction eventually produces $\mathrm{H}_{2}$ to store the photon energy in the form of chemical bonds, which can be regarded as artificial photosynthesis. Chromophores such as porphyrin and pyridine also serve as catalyzers. One of the significant similarities between Domcke's mechanism and ours is that conical intersections ${ }^{33}$ play important roles in splitting $\mathrm{OH}$ bonds.

Having mainly finished the study of the dynamical mechanism of photoinduced charge separation dynamics as above, we attempted to construct a chemical model of photocatalytic water splitting, inspired by the Kok cycle which is triggered by four-time photon absorption. After briefly describing the theoretical principles we used to construct the chemical model system in Sections 2.1 to 2.2, in Section 2.3 we propose a model reaction scheme that could materialize water splitting within a photon energy range of about $3 \mathrm{eV}$. In Section 3, we show the theoretical background from the viewpoint of nonadiabatic electron wavepacket dynamics for charge separation and feasibility of the concomitant chemical reactions from the viewpoint of energetics. We studied a possible mechanism for the formation of $\mathrm{O}_{2}$ molecules via water clusters contacting Mn-oxides. This paper concludes in Section 4 with some remarks. 


\section{Proposed reaction scheme of the water-splitting cycle}

We first present our studied mechanism of the photocatalytic water splitting cycle using small Mn oxides, from which oxygen molecules can be generated via contact with water clusters. This is one of the possible mechanisms that we surveyed and verified in terms of quantum mechanical calculations, including electron wavepacket dynamics and energetics of key temporal species. The actual study proceeds in a manner of "propose and verify" or trial and error to find appropriate processes, and hence a naive way of presentation according to the chronological order proceeds back and forth. Therefore, for the sake of systematic presentation we first summarize the attained cyclic reaction mechanism in this section, and verify the individual pieces of the reaction in greater detail in the next section.

\subsection{Charge separation dynamics triggering characteristic sequences of reactions}

2.1.1 Coupled proton electron-wavepacket transfers. The quantum mechanical mechanism of photoinduced charge separation by Mn oxides $\Omega$ is schematically summarized as: ${ }^{3,4}$

$$
\Omega-\mathrm{OH}_{2} \cdots \mathrm{A} \stackrel{h \nu}{\longrightarrow} \Omega^{\bullet}-\mathrm{OH} \cdots\left(\mathrm{H}^{\Delta+} \mathrm{A}^{\Delta-\bullet}\right)^{*}
$$

As an illustrative example system we chose $\Omega=\operatorname{MnCa}(\mathrm{OH})_{3}$ and $\mathrm{A}=$ intramolecular polarized 4-hydroxyaniline and imidazole (see Fig. 1). ${ }^{5}$ The symbols "...", “»", and "*” indicate a hydrogen bond, an unpaired electron (radical), and an excited electronic state, respectively. $\mathrm{H}^{\Delta+}$ indicates a proton with a partial positive charge to the extent of $0.5 e$, while $\mathrm{A}^{\Delta-\bullet}$ indicates an anion radical with the magnitude of the partial charge to the extent of $-0.5 e . \Omega$ denotes an arbitrary Mn oxide (complex), but was chosen to keep the system simple and stable. A serves as a proton-electron acceptor that has low-lying Rydberg-like excited states. One of the important characteristics of this mechanism is that the proton and electron take different pathways from each other to reach spatially different places in A. While the proton is accepted by a lone pair of the $\mathrm{N}$ atom of A just as in ground-state proton transfer, the electron is captured by a dense manifold of Rydberg-like diffused states of A to result in $\Delta \pm$ charge separation. This is a nonadiabatic process through conical intersections. This theoretically found mechanism has been widely identified in our studied photoinduced charge separation systems catalyzed by Mn oxides.

In this general model system, the proton and electron donor $\Omega-\mathrm{OH}_{2}$ is assumed to directly attach to the acceptor A. However, it is usual experimental practice for these systems to be dipped in bulk water, the inner part of which is structured by hydrogen-bonding networks. Barry, Brahmachari and Guo ${ }^{34}$ emphasize the critical importance of tracking reactive water in hydrogen-bonding networks in their recent review article about photosynthetic oxygen evolution in PSII. Moreover, the chemical bond $\Omega-O$ is strong enough to be maintained as it is throughout the excited dynamics as in eqn (4). It is therefore hard for the $\mathrm{OH}_{2}$ in $\Omega-\mathrm{OH}_{2}$ to be readily recycled for the system to repeat the reactions many times even if additional photons are provided. In other words, $\Omega-\mathrm{OH}_{2}$ in this setting cannot serve as a catalyst by itself. A natural idea to make a first step towards more flexible and general systems is to examine whether the proton transfer in eqn (4) is still possible through a relay channel, schematically suggested to be:

$$
\begin{aligned}
& \Omega-\mathrm{OH}_{2} \cdots \mathrm{OH}_{2} \cdots \mathrm{A} \stackrel{h \nu}{\longrightarrow} \\
& \Omega^{\bullet}-\mathrm{OH} \cdots \mathrm{OH}_{2} \cdots\left(\mathrm{H}^{\Delta+} \mathrm{A}^{\Delta-\bullet}\right)^{*}
\end{aligned}
$$

If possible, the water molecule in between the acceptor and donor can be linked to bulk water through another hydrogen bond. However, this is not a trivial question in that it is not obvious for electron transfer to take place efficiently as it did before since the distance between the donor and acceptor should become greater. Fortunately, this is indeed the case. We will show a concrete example of such nonadiabatic electron wavepacket dynamics of charge separation in Section 3.

\subsection{Photocatalytic cycle for water splitting and $\mathrm{O}_{2}$ generation}

The present study surveys (i) how the hydrogen-bonding networks can revive the $\Omega^{\bullet}-\mathrm{OH}$ in its excited state (recall eqn (4)) back to $\Omega-\mathrm{OH}_{2}$ in the ground state, thereby making it ready to absorb another photon to serve as a catalyst, and (ii) how oxygen molecules can be generated as a result of the series of reactions. We here propose a chemical model of the watersplitting cycle in which the system absorbs four photons to generate one molecule of oxygen. The mechanism is inspired by the Kok cycle (S-state cycle). ${ }^{35,36}$ We therefore refer to this bio-inspired simple mechanism as the "K-state cycle", $\mathrm{K}_{0} \rightarrow \mathrm{K}_{1}$, $\mathrm{K}_{1} \rightarrow \mathrm{K}_{2}, \mathrm{~K}_{2} \rightarrow \mathrm{K}_{3}$, and $\mathrm{K}_{3} \rightarrow \mathrm{K}_{0}$, the preview of which can be seen in Fig. 2. Along with the presentation of the details of the possible change of the states, we attempted to ensure that the involved reactions are energetically feasible.

2.2.1 Guiding principles to construct model chemical systems. The K-state cycle has been sought and designed as one of the possible systems that can be constructed under the following ideas.

1. A single photon excitation of the Mn oxides initiates a single charge separation and a concomitant series of reactions in the water network. The four photon process makes a single circuit of the catalytic cycle. Each photoinduced charge separation dynamics takes place with the same CPEWT mechanism under a common optical source.

2. The electron and proton thus isolated by the photoinduced charge separation dynamics are supposed to be carried away to their own destinations, and will not come back to the Kstate system.

3. The four individual subprocesses, $K_{0} \rightarrow K_{1}, K_{1} \rightarrow K_{2}$, $\mathrm{K}_{2} \rightarrow \mathrm{K}_{3}$, and $\mathrm{K}_{3} \rightarrow \mathrm{K}_{0}$, should share as many common elementary processes as possible. This mimics a kind of parsimony principle frequently found in biological systems, in which molecules and/or sets of molecular systems are utilized multiple times for different purposes so that the system is kept as compact and economical as possible. 

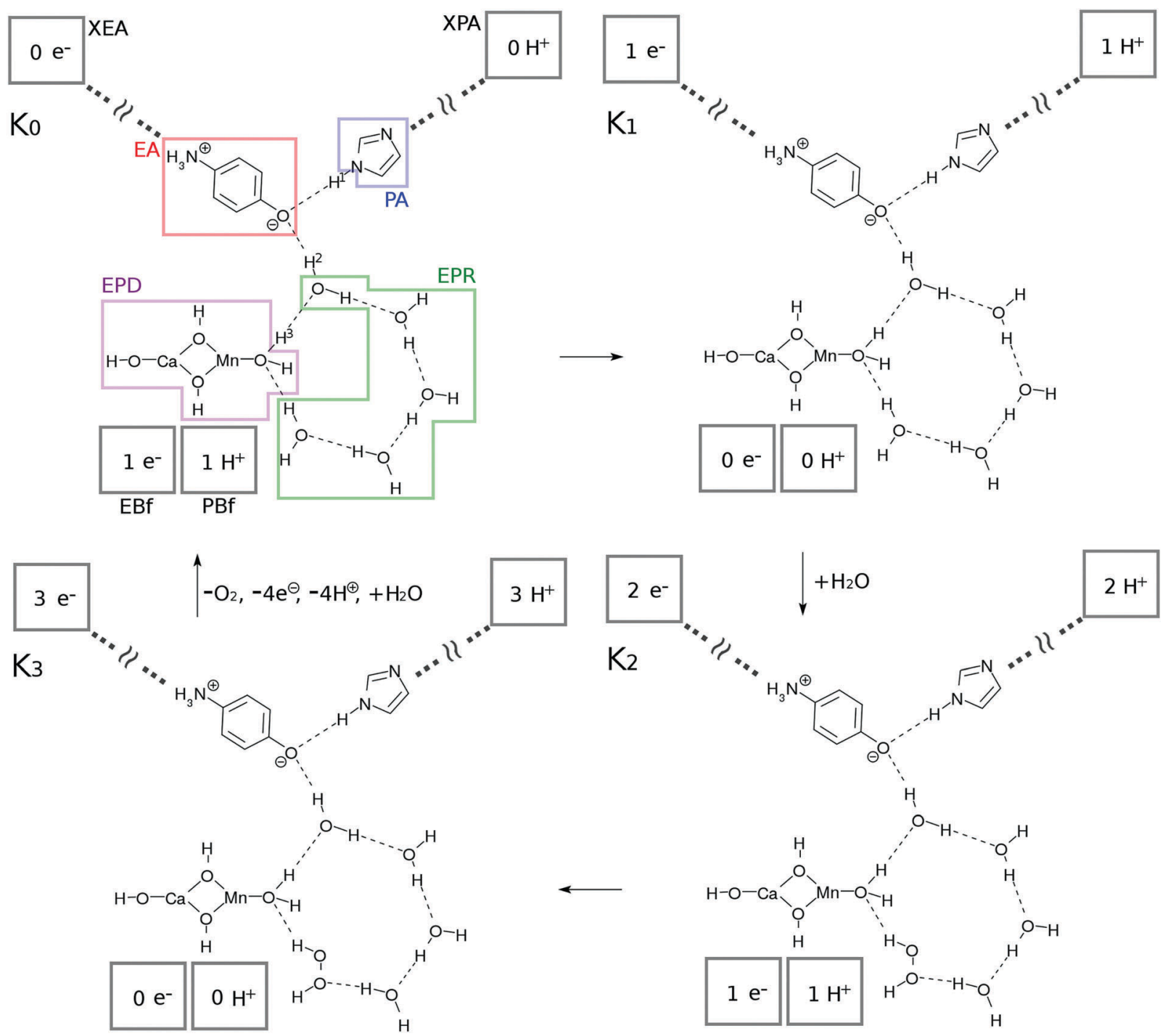

Fig. 2 Schematic representation of the present photocatalytic water-splitting cycle. This cycle has four states referred to as "K-states" and each is triggered by CPEWT. See text for the labels and components involved.

4. The system should use materials and molecules that are available abundantly. Here, water is used.

2.2.2 System composition and basic units from which to start photoinduced dynamics

Subsystems and their functions. We first show the basic units, $\mathrm{K}_{0}$, $\mathrm{K}_{1}, \mathrm{~K}_{2}$, and $\mathrm{K}_{3}$ in Fig. 2, from which the four-photon catalytic reaction makes one complete cycle. Each of these is in its ground state and is waiting to absorb light. Upon excitation, the individual series of reactions follow the pathway shown in the next subsection.

The catalytic system commonly consists of subsystems, namely, an electron-proton donor $\left(\mathrm{EPD}=\mathrm{MnCa}(\mathrm{OH})_{4}\right)$, an electron acceptor $\left(\mathrm{EA}=\mathrm{O}-\mathrm{C}_{6} \mathrm{H}_{4}-\mathrm{NH}_{3}\right)$, a proton acceptor $(\mathrm{PA}=$ $\mathrm{C}_{3} \mathrm{~N}_{2} \mathrm{H}_{3}$ ), electron-proton resources $\left(\mathrm{EPR}=\mathrm{OH} \cdots\left(\mathrm{H}_{2} \mathrm{O}\right)_{3} \cdots \mathrm{X}\right.$, $\mathrm{X}=\mathrm{H}_{2} \mathrm{O}$ for $\mathrm{K}_{0}, \mathrm{~K}_{1}$ and $\mathrm{H}_{2} \mathrm{O}_{2}$ for $\mathrm{K}_{2}, \mathrm{~K}_{3}$ ), and electron and proton buffering subsystems (EBf and PBf, respectively). Their chemical roles will be described in order.

This system is open (dissipative) to the surrounding environment; two water molecules are taken in from the outside while 4 protons,
4 electrons and one oxygen molecule are produced and transported out of the catalytic system.

Protons and electrons are transported to asymptotic sites. As $\mathrm{K}_{n}$ proceeds by one step $(0 \rightarrow 1 \rightarrow 2 \rightarrow 3 \rightarrow 0)$, the electron and proton created in the photoinduced charge separation are eventually transported to XEA through EA, and XPA through PA, respectively (see Fig. 2). Thus the numbers of electrons and protons in XEA and XPA, respectively, increase one by one. XEA is defined to abstract the electron delivered to the Rydberg-like states of the EA as XEA- $\mathrm{e}^{-}$and is supposed to serve as an electron carrier like the quinones in PSII. ${ }^{1,2}$ The explicit mechanism of the electron abstraction is not addressed in the present paper, but its energy is considered later in the study of the energetics. Note that the XEA site has the capacity to accept electrons (modulo 4) after each photocatalytic cycle. XPA is similarly defined to abstract the proton of PA to become $\mathrm{XPA}-\mathrm{H}^{+}$. 
Hydrogen-bonding network. As seen in Fig. 2, there is a water molecule $\left(\mathrm{H}^{2} \mathrm{OH}\right.$ in $\mathrm{K}_{0}$ of Fig. 2) in between EPD and EA linking them via hydrogen bonding. Notice that this particular water molecule was not considered in our former model of photoinduced charge separation dynamics, which is shown in Fig. 1. Thanks to this water, the charge separation center (EPD, EA, and PA units) is now attached to other water molecules, which constitutes a water cluster. Here we consider a six-membered cluster, since it is generally believed to stably exist in bulk water. However, our preliminary calculations show that the fivemembered cluster works as well for $\mathrm{O}_{2}$ generation.

The system shown in Fig. 1 has been naturally extended so as to be electronically connected with the bulk water surrounding the charge separation site. Thus water molecules consumed in water splitting as in eqn (2) can be readily supplied and are now recyclable. The cost is the possibility of the breakdown of CPEWT through the mediating water molecule. Our critical computational finding is that photoinduced charge separation under the CPEWT mechanism is indeed possible in this system too, which will be explicitly shown in Section 3 .

The system includes three hydrogen atoms which are involved in the proton relay-transfer of the CPEWT, the initial configuration of which is

$$
\text { EPD- } \mathrm{H}^{3} \cdots \text { EPR-H }{ }^{2} \cdots \text { EA } \cdots \mathrm{H}^{1}-\mathrm{PA}
$$

in which “..." indicates hydrogen-bonding. The labels $\mathrm{H}^{n}$ $(n=1,2,3)$ are used to distinguish them from other nonreactive hydrogen atoms (see Fig. 2). $\mathrm{H}^{1}$ is already transferred to the PA in the ground state before the photoinduced charge separation begins because of an external condition to pull a proton towards XPA. ${ }^{5}$ This is regarded as one of the relay proton transfers to effectively carry a proton from the EPD to the PA.

Electron- and proton-buffering in the catalytic cycle. Photoinduced charge separation and proton transfer through the configuration change as in eqn (6) should strongly affect the water cluster that is directly attached to the mediating water molecule. We here assume that different kinds of proton- and electron-transfers are induced in this water cluster, through which oxidation-reduction reactions and charge neutralization reactions take place in the water cluster too. Since the generated $\mathrm{O}_{2}$ is released (exhausted) asymptotically, another water molecule should be added into the cluster. For these reactions to proceed in low energy, we require the presence of buffering systems (molecules) that can store and release electrons and protons such as some amino acid residues (e.g. tyrosine and tryptophan ${ }^{37-41}$ ) or quinones in PSII. ${ }^{1,2}$ For instance, an electron buffer (EBf) captures electrons as EBf- $\mathrm{e}^{-}$, while $\mathrm{EBf}-\mathrm{e}^{-}$releases an electron as $\mathrm{EBf}+\mathrm{e}^{-}$. A similar relationship exists for proton buffer $\mathrm{PBf}$ and $\mathrm{PBf}-\mathrm{H}^{+}$in response to a change of environment.

The EBf is supposed to have two roles, namely, giving an electron to the EPD in the $\mathrm{K}_{0}-\mathrm{K}_{1}$ and $\mathrm{K}_{2}-\mathrm{K}_{3}$ transitions, and abstracting an electron from $\mathrm{H}_{2} \mathrm{O}$ (the $\mathrm{K}_{1}-\mathrm{K}_{2}$ transition) or $\mathrm{H}_{2} \mathrm{O}_{2}$ (the $\mathrm{K}_{3}-\mathrm{K}_{0}$ transition) of the EPR. The PBf has similar roles, exchanging protons from and to the cluster. In the present model setting, we do not specify the molecular species for EBf and PBf. However, the energetics with respect to $\mathrm{PBf} \leftrightarrow \mathrm{PBf}-\mathrm{H}^{+}$ and EBf $\leftrightarrow$ EBf- $\mathrm{e}^{-}$will be discussed later in Section 3.4.

2.2.3 Gross products after one cycle. In the K-state cycle of Fig. 2, the first two photons, namely those absorbed by $\mathrm{K}_{0}$ and $\mathrm{K}_{1}$, are used in a reaction to form hydrogen peroxide, $\mathrm{H}_{2} \mathrm{O}_{2}$, from two water molecules via:

$$
2 \mathrm{H}_{2} \mathrm{O}+2 h \nu \rightarrow \mathrm{H}_{2} \mathrm{O}_{2}+2 \mathrm{H}^{+}+2 \mathrm{e}^{-}
$$

Notice that the configurations of the EPD, EA, PA, and water cluster in $K_{1}$ remain the same as those in $K_{0}$. One of the water molecules of the cluster is substituted with hydrogen peroxide, $\mathrm{H}_{2} \mathrm{O}_{2}$, in the $\mathrm{K}_{1}-\mathrm{K}_{2}$ transition, and $\mathrm{H}_{2} \mathrm{O}_{2}$ and $\mathrm{O}_{2}$ are generated within this water cluster. Thus eqn (7) gives a collective representation of the $\mathrm{K}_{0}-\mathrm{K}_{1}$ and $\mathrm{K}_{1}-\mathrm{K}_{2}$ transitions. Note that the $\mathrm{H}_{2} \mathrm{O}_{2}$ molecule is formed within the EPR (water cluster). The third and fourth photons are used in the reactions of $\mathrm{K}_{2}-\mathrm{K}_{3}$ and $\mathrm{K}_{3}-\mathrm{K}_{0}$ to generate an $\mathrm{O}_{2}$ molecule from the $\mathrm{H}_{2} \mathrm{O}_{2}$, via:

$$
\mathrm{H}_{2} \mathrm{O}_{2}+2 h \nu \rightarrow \mathrm{O}_{2}+2 \mathrm{H}^{+}+2 \mathrm{e}^{-}
$$

This step corresponds to the $K_{2}-K_{3}$ and $K_{3}-K_{0}$ transitions. Water-splitting by means of eqn (2) is thus obtained by combining eqn (7) and (8).

\subsection{Reactions following each photoexcitation}

After the charge separation dynamics occur upon photoexcitation of the $\mathrm{Mn}$ oxide in each K-state, characteristic reactions follow in the water cluster and the electron and proton buffers. Each elementary step of them is denoted as $K_{n m}(m=1,2,3 \ldots)$, where the suffix $n$ distinguishes the four K-states $\left(K_{0}\right.$ to $\left.K_{3}\right)$ and $m$ identifies the sequence of reactions after each photoexcitation. For instance, once $K_{0}$ is excited, the reactions $K_{01}$ to $K_{16}$ follow ( $\mathrm{K}_{01}$ to $\mathrm{K}_{03}$ are drawn in Fig. 3, while $\mathrm{K}_{04}$ to $\mathrm{K}_{1}$ are depicted in Fig. 4). The maximum number of $m$ depends on $n$. For instance, $K_{1}$ has a maximum of $K_{16}$ whereas $K_{04}$ is the last for $\mathrm{K}_{0}$.

2.3.1 Common steps included in $K_{n m}(m=1-4)$. Two states $\mathrm{K}_{n m}$ with different $n$ are similar to each other if $m$ is the same. In particular, the configurations and their change with respect to electron and proton transfers from the $\mathrm{Mn}$ oxide to EA (eventually to XEA) and PA (eventually to XPA) through the mediating water molecule are the same with respect to $n$ for a given $m$ from $m=1$ up to 4 . The time-evolution in this stage is depicted in Fig. 3.

More precisely, the first process $\left(\mathrm{K}_{n}-\mathrm{K}_{n 1}\right)$ simply represents the elementary process of CPEWT, which is followed by electron transport to XEA (in $\mathrm{K}_{n 1}$ ) and proton relay transport (in $\mathrm{K}_{n 2}-\mathrm{K}_{n 3}$ ). The physical timing of these two transports is not specified here and should depend on a system under study.

The dynamics of CPEWT will be discussed in greater detail in Section 3. We do not address the electron abstraction mechanism from EA to XEA, but only the energetics are taken into account to confirm that the reaction is indeed possible. There are several mechanisms for the proton to be handed from the PA to the channel connected to the XPA. One possible way is a rotation of the PA. This motion will be accompanied by 

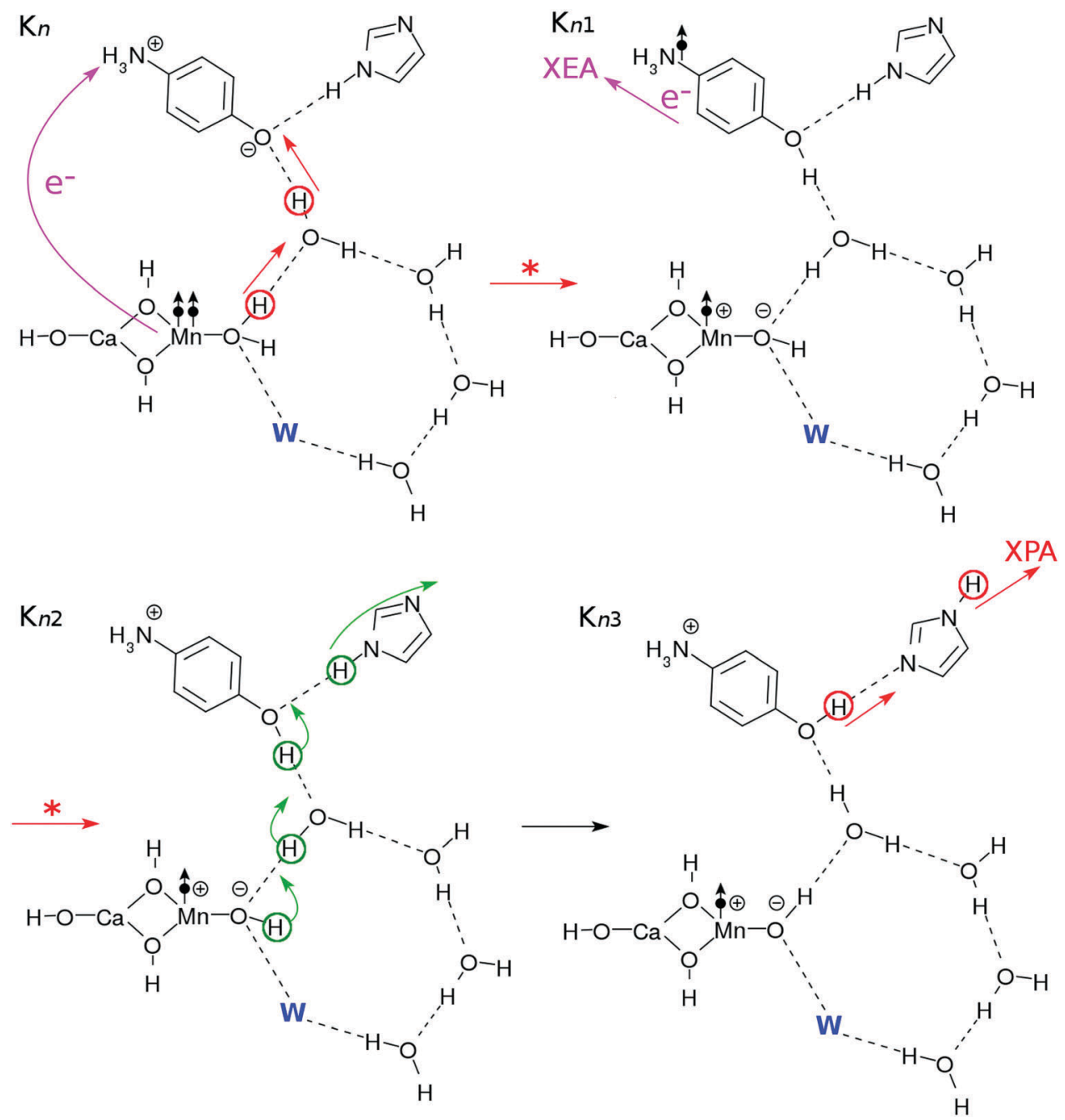

Fig. 3 Reaction schemes commonly involved in the $K_{n}-K_{n+1}$ transition $\left(n=0,1,2\right.$ or 3 with $\left.K_{4}=K_{0}\right)$, in which abstraction of an electron and a proton from "the core subsystem" take place. The atoms that are responsible for each reaction are indicated by circles and arrows (the straight ones schematically indicate transfer and the curly ones represent reorganization of the hydrogen bonds). The electron transfers are indicated by the arrows with " $\mathrm{e}^{-"}$. The "W" (blue bold letter) indicates a substitute, which is $\mathrm{H}_{2} \mathrm{O}$ for $n=0$ or 1 and $\mathrm{H}_{2} \mathrm{O}_{2}$ for $n=2$ or 3 . The reactions in the electronic excited states are indicated by arrows with "*". The dot-and-arrow symbols schematically indicate the locations of radicals.

reorganization of the hydrogen-bond network. In the fourth step $\left(\mathrm{K}_{n 3}-\mathrm{K}_{n 4}\right)$, the proton facing the external system is abstracted by the XPA. It involves proton transfer from the EA to the PA.

The processes resuming from $\mathrm{K}_{n 4}$ depend on $n$, as will be discussed next. This is because the configurations of the hydrogen-bonding network, PBf and EBf are different from one another, thereby distinguishing the evolutions of $\mathrm{K}_{n}$ $(n=0-3)$.

2.3.2 $K_{\mathbf{0}}-K_{1}$ transition. The subprocesses from $K_{0}$ to $K_{03}$ have been described as above and in Fig. 3. The dynamics up to this stage are concerned with electron and proton transfers, which start from the photoexcited separation. A new event takes place in $\mathrm{K}_{04}$ to $\mathrm{K}_{1}$, as illustrated in Fig. 4. After the protons and electrons are isolated and transported by photoexcitation of $\mathrm{K}_{0}$, the $\mathrm{Mn}$ oxides are left polarized (schematically represented as $\mathrm{K}_{04}$ in Fig. 4). To compensate for the lost charges due to the missing electron and proton, the relevant sites are neutralized by accepting electrons and protons from the individual buffers. This brings the configurations of the core subsystem (Mn-oxide, water cluster, EA, and PA) back to the exact same form of $\mathrm{K}_{0}$ in its ground state. Note, however, that the surroundings of the core part in $\mathrm{K}_{1}$ are now different from those of $\mathrm{K}_{0}$ in that $\mathrm{EBf}-\mathrm{e}^{-}$and $\mathrm{PBf}-\mathrm{H}^{+}$ have turned out to be EBf and PBf, respectively.

2.3.3 $\quad K_{1}-K_{2}$ transition forming $\mathbf{H O O H}$. The core subsystem (Mn-oxide, water cluster, EA, and PA) involved in the $\mathrm{K}_{1}$ system is ready to absorb the second photon to resume the second charge separation. The subprocess that the core subsystem experiences, in which CPEWT led to $K_{1}-K_{11}-K_{12}-K_{13}$, is similarly represented in Fig. 3. However, since we now have EBf and $\mathrm{PBf}$ in place of $\mathrm{EBf}-\mathrm{e}^{-}$and $\mathrm{PBf}-\mathrm{H}^{+}$, respectively, the charge neutralization for the Mn-oxide can proceed as schematically illustrated in Fig. 5: after $\mathrm{K}_{13}$, one of water molecules in the cluster gives an electron from one of its lone pairs to EBf to 


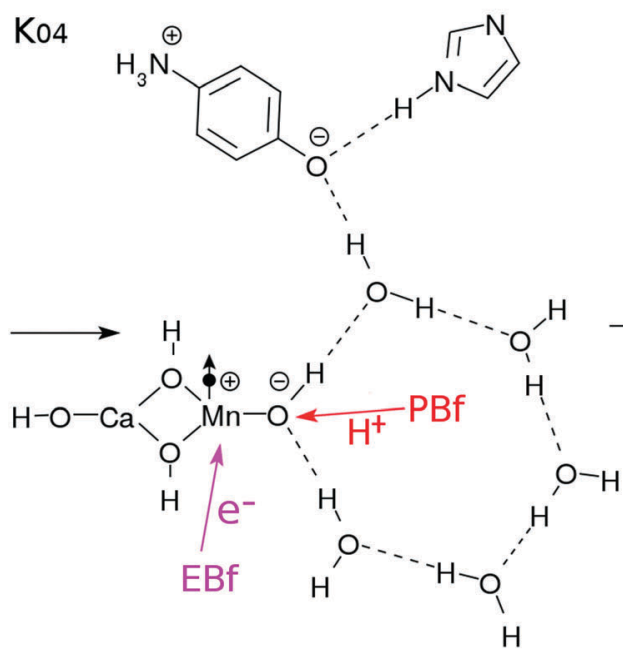

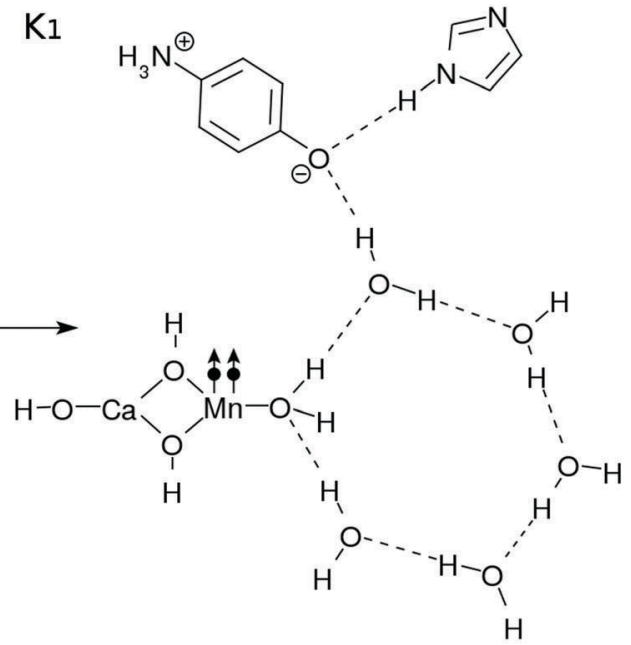

Fig. 4 Reaction schemes involved in the $K_{0}-K_{1}$ transition. The electron transfers are indicated by the arrows with "e $e^{-"}$.

form $\mathrm{EBf}-\mathrm{e}^{-}$, and simultaneously or successively donates protons to $\mathrm{PBf}$ making $\mathrm{PBf}-\mathrm{H}^{+}$(see $\mathrm{K}_{14}$ in Fig. 5). The relevant water molecule is then deprotonated and looks like ${ }^{\bullet} \mathrm{OH}$ (neutral radical). Next, the zwitter ion on $\mathrm{Mn}-\mathrm{O}$ generated after photoexcitation is neutralized by a nearby water molecule by accepting protons and electrons. Thus another tentative ${ }^{\bullet} \mathrm{OH}$ is created (see $\mathrm{K}_{15}$ in Fig. 5). Then, these two tentatively-formed - OHs combine with each other to form $\mathrm{HOOH}$, which stays around under the support of hydrogen-bonding ( $\mathrm{K}_{16}$ in Fig. 5). The reaction mechanism of $\mathrm{HOOH}$ formation is discussed in
K14

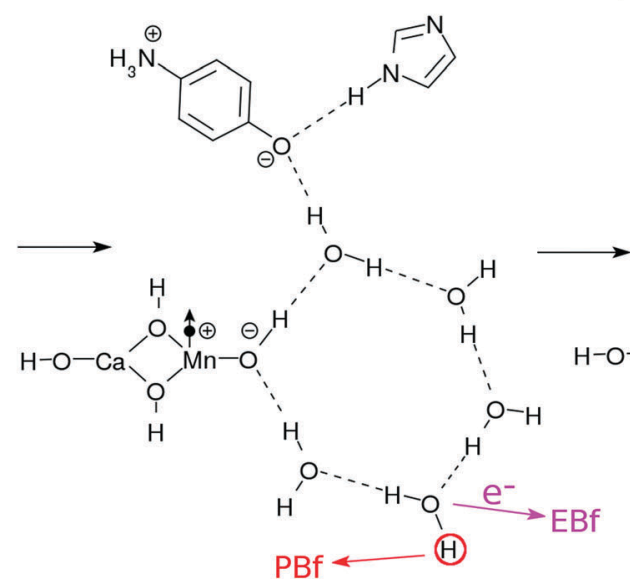

$\mathrm{K} 15$<smiles>[O-]c1ccc(OCCn2ccnc2)cc1</smiles>

H

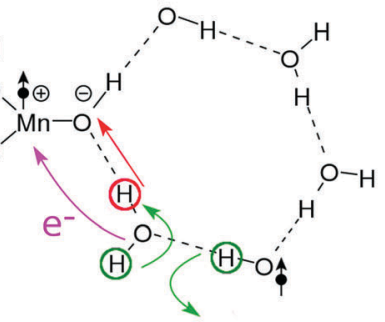

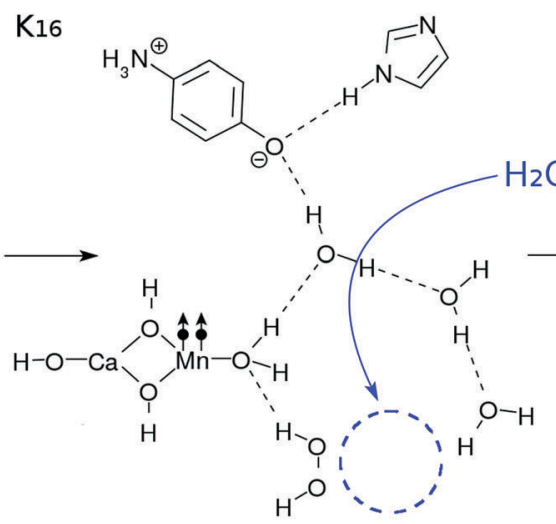

$\mathrm{K} 2$<smiles></smiles><smiles>CC(C)C</smiles><smiles>C1CCOCC1</smiles>

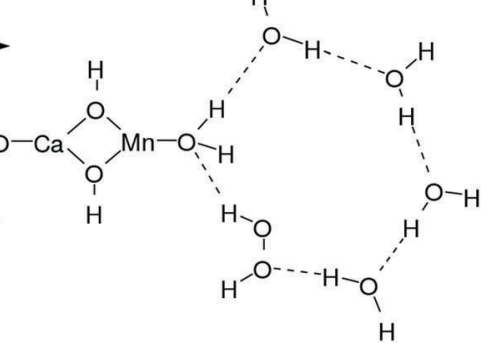

Fig. 5 Reaction schemes involved in the $K_{1}-K_{2}$ transition. The electron transfers are indicated by the arrows with " $e^{-"}$. 
detail in Section 3.5. These processes proceed in a concurrent manner, rather than successively, thereby avoiding possible energy dissipation.

Two protons and two electrons are lost from the core subsystem of the $\mathrm{K}_{0}$ state, leaving $\mathrm{HOOH}$ behind. This implies that a vacancy in the water-cluster is made. To fill this vacancy in order to maintain the stability of the hydrogen-bonding network, a water molecule may be supplied from other surrounding places of bulk water to restore the network. This configuration is depicted in $K_{2}$ in Fig. 5, which is in the ground state, awaiting the third photoexcitation.

2.3.4 $\mathbf{K}_{2}-\mathbf{K}_{3}$ transition. Upon excitation due to the third photoabsorption, the $K_{2}$ state proceeds to $K_{3}$ through $K_{2}-K_{23}$ (Fig. 3 with $\mathbf{W}$ being $\mathrm{HOOH}$ ) and $\mathrm{K}_{24}-\mathrm{K}_{3}$ (Fig. 6). Once again the charge neutralization on $\mathrm{Mn}-\mathrm{O}$ in the Mn-oxide takes place as in the $\mathrm{K}_{04}-\mathrm{K}_{1}$ of Fig. 4 . The major difference is found only in the presence of $\mathrm{H}_{2} \mathrm{O}_{2}$ instead of $\mathrm{H}_{2} \mathrm{O}$ in the water cluster. As a result, EBf and PBf remain in the $K_{3}$ system (see $K_{3}$ in Fig. 2).

2.3.5 $\mathrm{K}_{3}-\mathrm{K}_{0}$ transition yielding $\mathrm{O}=\mathrm{O}$ and making the cycle complete. As shown in Fig. 7 , the $K_{3}-K_{0}$ transition takes place through three steps triggered by photoexcited CPEWT due to the fourth photon (Fig. 3). The essential steps needed to complete the cycle, in which another water molecule is supplied from the bulk water and $\mathrm{O}_{2}$ is left behind, are schematically illustrated in Fig. 7. The series of elementary processes here are rather similar to those in the transitions of $\mathrm{K}_{14}-\mathrm{K}_{2}$ of Fig. 5 .

In the step $\mathrm{K}_{34}-\mathrm{K}_{35}$, the EBf and the PBf effectively abstract the electron and the proton from the $\mathrm{H}_{2} \mathrm{O}_{2}$ molecule, respectively, to generate a tentative hydroperoxyl radical $\mathrm{OOH}^{\bullet}$. In the step $\mathrm{K}_{35}-\mathrm{K}_{36}$, another proton and electron transfer from $\mathrm{OOH}^{\bullet}$ to the Mn-oxide site to neutralize the zwitter-ion pair (see $K_{35}$ ). Once again these two elementary processes are likely to take place coherently. After all these transfers, an $\mathrm{O}=\mathrm{O}$ bond in spin triplet is released. The energetics of this reaction mechanism will be discussed in detail in Section 3.5.

Upon formation of the oxygen molecule, the hydrogenbonding network can be disconnected and destabilized. Therefore, a supply of a water molecule is made possible as in $K_{36}$, and the configuration of the core subsystem in $K_{0}$ is restored, including those of the buffers $\mathrm{EBf}-\mathrm{e}^{-}$and $\mathrm{PBF}-\mathrm{H}^{+}$.

\section{Charge separation dynamics and energetics behind the reaction mechanisms proposed: theoretical background}

This section is devoted to showing the theoretical foundation of the proposed mechanism of the photocatalytic cycle of water splitting. Its background to verify the mechanism consists of two aspects: one is the photo-induced charge separation dynamics (coupled proton electron-wavepacket transfer (CPEWT)) studied by means of nonadiabatic electron wavepacket dynamics. The other is energetics with respect to the characteristic structure of molecular systems and their associated energies. We here assure that all of the reaction processes following the four individual photoexcitations can proceed within the tolerance of about $3 \mathrm{eV}$. Solvent effects due to the bulk water and possible protein moiety are not considered because of computational limitations.

\subsection{The theory of nonadiabatic electron wavepackets}

We briefly outline the theory of nonadiabatic electron-wavepacket dynamics, which plays an important role in characterizing the present mechanism. The full-dimensional nonadiabatic electronwavepacket dynamics demonstrated herein are performed via the semiclassical Ehrenfest theory (SET). ${ }^{42-46}$ The SET is derived as an approximation to the path-branching representation theory. ${ }^{4-50}$ See ref. 4 for the path-branching dynamics on the relevant smaller systems. Here we only give a brief review of the path-branching representation and the derivation of the SET. See ref. 3-5 and 51-62 for applications. In this theory, electron dynamics is described in terms of the quantum wavepacket to be evolved in time along nuclear branching paths. The electron-wavepacket $\Psi_{\text {elec }}(\mathbf{r}, t ; \mathbf{R}(t))$ is expanded in a series of time-independent

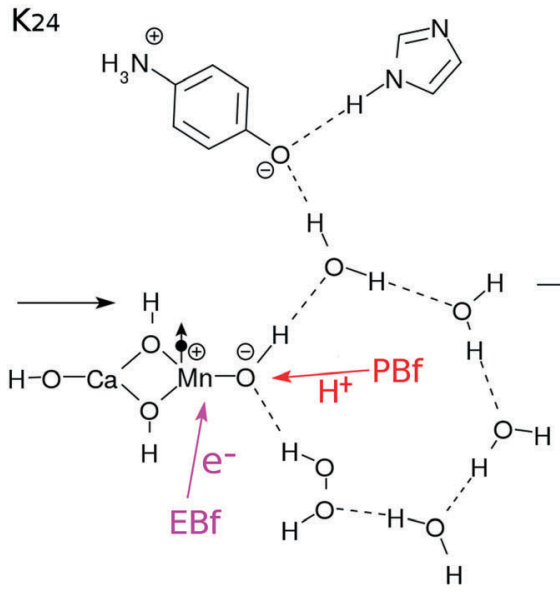

$\mathrm{K} 3$

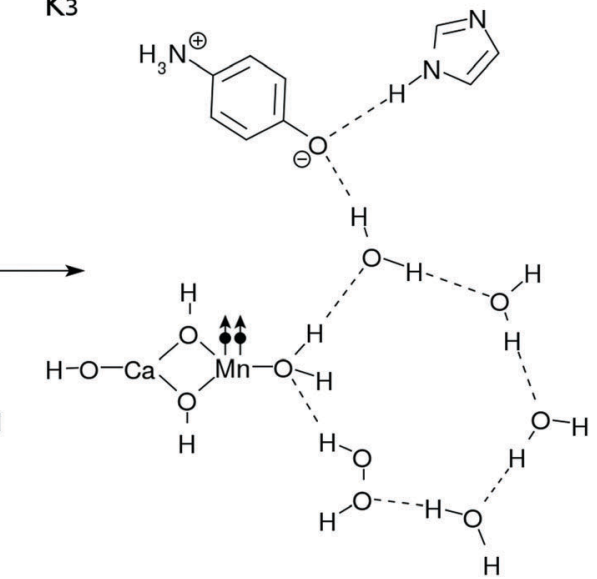

Fig. 6 Reaction schemes involved in the $K_{2}-K_{3}$ transition. 


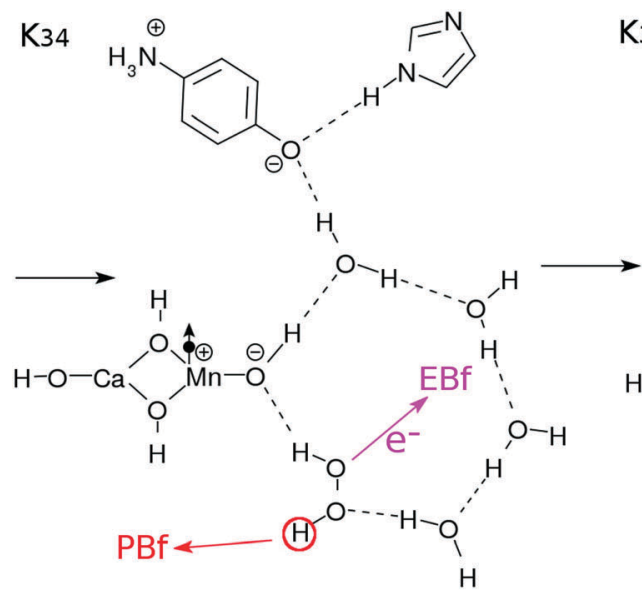

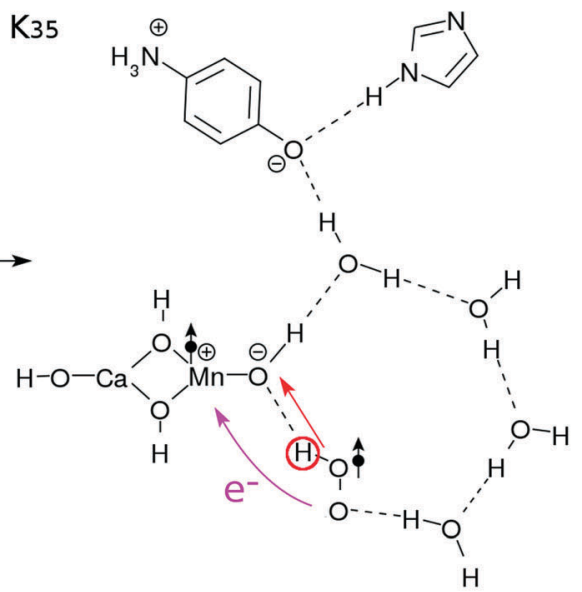

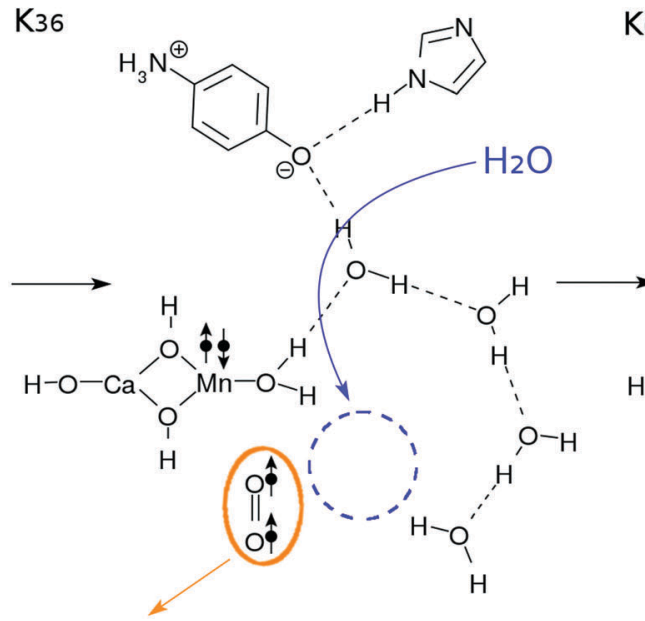

Ko<smiles>[NH3+]c1ccc(OCCn2ccnc2)cc1</smiles>

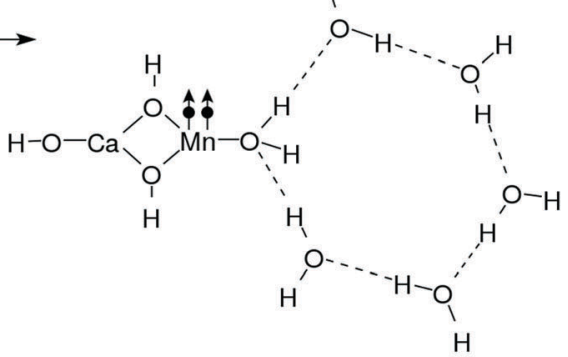

Fig. 7 Reaction schemes involved in the $K_{3}-K_{0}$ transition. The electron transfers are indicated by the arrows with " $e^{-"}$. These steps finally bring this system back to the $K_{0}$ state.

wavefunctions $\left\{\Phi_{I}(\mathbf{r}, \mathbf{R})\right\}$ that are parameterized with nuclear geometry $\mathbf{R}$ at time $t$ as in

$$
\Psi_{\text {elec }}(\mathbf{r}, t ; \mathbf{R}(t))=\left.\sum_{I} C_{I}(t) \Phi_{I}(\mathbf{r}, \mathbf{R})\right|_{\mathbf{R}=\mathbf{R}(t)}
$$

in which $\left\{C_{I}(t)\right\}$ is a set of time-dependent coefficients to be determined, and $\mathbf{r}$ denotes the coordinates of all of the electrons involved. One may employ any type of orthonormal basis functions as $\left\{\Phi_{I}(\mathbf{r}, \mathbf{R})\right\}$ including Slater determinants, configuration state functions (CSFs), and adiabatic states. The time evolution of the electron-wavepacket follows the equation of motion expressed as

$$
i \hbar \frac{\mathrm{d} C_{I}}{\mathrm{~d} t}=\sum_{J}\left[H_{I J}^{(\mathrm{el})}-i \hbar \sum_{k} \dot{R}_{k} X_{I J}^{k}-\frac{\hbar^{2}}{4} \sum_{k}\left(Y_{I J}^{k}+Y_{J I}^{k *}\right)\right] C_{J}
$$

in which the relevant matrix elements are represented as

$$
\begin{aligned}
H_{I J}^{(\mathrm{el})} & =\left\langle\Phi_{I}\left|\hat{H}^{(\mathrm{el})}\right| \Phi_{J}\right\rangle, \quad X_{I J}^{k}=\left\langle\Phi_{I}\left|\frac{\partial}{\partial R_{k}}\right| \Phi_{J}\right\rangle, \\
Y_{I J}^{k} & =\left\langle\Phi_{I}\left|\frac{\partial^{2}}{\partial R_{k}^{2}}\right| \Phi_{J}\right\rangle
\end{aligned}
$$

Here $\hat{H}^{\text {(el) }}$ denotes the electronic Hamiltonian. Note that the scalar product in terms of the bra-ket notation in the present paper denotes the integration in terms of electronic coordinates. The terms multiplied by $\hbar^{2}$ in the right hand side of eqn (10) are the nontrivial correction to the conventional SET regarding electrons, but are usually neglected because of the presence of the small quantity $\hbar^{2}{ }^{61}$ The nuclear path is driven by the force matrix $F_{I J}^{k 47-50}$ expressed as:

$$
\begin{aligned}
F_{I J}^{k}= & -\left[\frac{\partial H_{I J}^{(\mathrm{el})}}{\partial R_{k}}+\sum_{K}\left(X_{I K}^{k} H_{K J}^{(\mathrm{el})}-H_{I K}^{(\mathrm{el})} X_{K J}^{k}\right)\right] \\
& +i \hbar \sum_{l} \dot{R}_{l}\left[\frac{\partial X_{I J}^{l}}{\partial R_{k}}-\frac{\partial X_{I J}^{k}}{\partial R_{l}}\right]
\end{aligned}
$$

Path-branching is induced by the off-diagonal elements of $F_{I J}^{k}$ at each small time step. The infinite number of path-branching is necessary to obtain the exact solution. Various methods exist to avoid such computational difficulty. As the drastically simple approximation, the SET takes an average of the force matrix over 
a single electronic wavepacket in such a way that:

$$
\begin{aligned}
\left\langle F_{k}\right\rangle= & \sum_{I J} C_{I}^{*} F_{I J}^{k} C_{J}=-\sum_{I J} C_{I}^{*} \frac{\partial H_{I J}^{(\mathrm{el})}}{\partial R_{k}} C_{J} \\
& -\sum_{I J K} C_{I}^{*}\left(X_{I K}^{k} H_{K J}^{(\mathrm{el})}-H_{I K}^{(\mathrm{el})} X_{K J}^{k}\right) C_{J},
\end{aligned}
$$

which can be rewritten as

$$
\left\langle F_{k}\right\rangle=-\left\langle\Psi_{\text {elec }}(\mathbf{r}, t ; \mathbf{R}(t))\left|\frac{\partial \hat{H}^{(\mathrm{el})}}{\partial R_{k}}\right| \Psi_{\text {elec }}(\mathbf{r}, t ; \mathbf{R}(t))\right\rangle,
$$

when the basis set $\left\{\Phi_{I}(\mathbf{r}, \mathbf{R})\right\}$ is complete.

\subsection{Computational details}

The atomic basis set chosen is basically common to all of the calculations performed in what follows, although different levels of computation are taken for different purposes. We employ the Stevens, Basch, Krauss, Jasien, and Cundari effective core potentials (SBKJC ECPs) ${ }^{63}$ for Mn and Pople's 6-31G for the other atoms. We add diffuse orbitals to the $\mathrm{N}$ and $\mathrm{H}$ $(6-31++G)$ atoms of the EA. The diffuse orbitals are crucial in order to describe the present mechanism involving the dense manifold of the Rydberg-like states. ${ }^{3-5,64}$ All of the quantum chemical calculations are performed with use of the GAMESS quantum chemistry package, ${ }^{65,66}$ in which our original codes have been implemented to carry out the nonadiabatic electron wavepacket dynamics. For the geometry optimization to locate the stationary point geometry, we used the restricted (either closed-shell or open-shell) Hartree-Fock (RHF) method. Otherwise, the electronic wavepackets are represented in terms of linear combinations of many electronic configurations up to single and double excitations.

\subsection{CPEWT in triplet states involving a hydrogen-bond network}

To track the dynamics we refer to the common core system of $K_{0}$ and $K_{1}$ as D11, whereas that of $K_{2}$ and $K_{3}$ as D21 (see Fig. 8). We first show numerically that the CPEWT previously found in simpler singlet systems ${ }^{3-5}$ also works in the present systems like D11 in a similar manner. Two major differences from what we did before are that the present systems have recyclable water molecules in the hydrogen-bond network, and they are spin triplet. In particular, it is far from obvious whether the CPEWT works in the presence of a hydrogen-bonding network, through which proton transfer can be driven along with electron transfer in different paths. Incidentally, a similar mechanism has been confirmed for the spin singlet counterpart as well.

We employ the CISD/RHF (triplet) level of calculations and use the low-lying 150 adiabatic states for the wavefunction $\left\{\Phi_{I}\right\}$ in eqn (10), in which CISD stands for configuration interaction of single and double excitations. The CISD active space is chosen as follows: the two SOMOs are employed to be the active MOs, while LUMO-(LUMO+89) are designated to be the active virtual MOs. All of the doubly occupied MOs and MOs higher than LUMO+89 are set to be inactive (frozen). The number of configuration state functions (CSFs) turns out to be 4186 . We employ the triplet RHF for the geometry optimization only, since the ground state of this system in the CISD level calculations around the optimized geometry is known to be dominated by the RHF configuration.

Electron dynamics calculations with CISD/RHF in triplet state generally require multi-reference CI, in which multiple spin functions to describe the triplet are taken into account. If therefore we start from a single reference to propagate the wavepacket in CI, we often face discontinuity in the choice of the most appropriate spin function. This is because different single reference functions can give birth to (slightly) different sets of molecular orbitals. To circumvent this technical difficulty, multi-reference methods should be appropriately handled in such regions. However, this method costs much computational time and labor, and we connect the electron-wavepackets expanded in different reference sets of MOs at a path-point(s) of disconnection, so as to maximize the overlap of the electron-wavepackets. See Section S1 (ESI $\dagger$ ) for the technical details.

3.3.1 Characteristic molecular orbitals and energy levels. As shown in Fig. 9, we find close similarities between molecular orbitals and associated energy levels that constitute D11 and D22 at their optimized geometries. In particular, one of the singly-occupied MOs (SOMOs) is dominated by the Mn d-shell $(\operatorname{SOMO}(1))$, and the other SOMO $(\operatorname{SOMO}(2))$ is dominated by the superposition of the $\mathrm{Mn} \mathrm{s-}$ and d-shells. The excited-state configurations associated with $\mathrm{SOMO}(1)$ make only little contribution to the low-lying excited states, whereas $\operatorname{SOMO}(2)$ is responsible for them. Calculations of the oscillator strength (Section S1 (ESI $\dagger$ )) show that some of those relatively low-lying excited states can be accessed by $\operatorname{SOMO}(2)$ by photoexcitation. We note that the lowest unoccupied MO (LUMO) and LUMO+1 are the Rydberg-like diffused states, which eventually serve as an electron acceptor (EA) in the CPEWT mechanism. However, such electron transfer to these Rydberg-like states is not made possible by direct photoexcitation, and can be realized only by nonadiabatic transition induced by the associated proton transfer passing through nearby conical intersections. ${ }^{3-5}$

3.3.2 Initial conditions for the nonadiabatic electron dynamics. In running the nonadiabatic electron wavepacket dynamics, we adopt rather simplified nuclear initial conditions compared to those of the previous work in which nuclear initial configurations are randomly taken from the ground-state path corresponding to the zero-point vibration. ${ }^{5}$ More precisely, in both the cases of D11 and D21, the initial nuclear positions are set to be the optimized geometry in the electronic ground state. The initial nuclear momenta are given only for the $\mathrm{H}^{n}$ ( $n=2$ or 3 ). The kinetic energies given to the $\mathrm{H}^{2}$ and $\mathrm{H}^{3}$ atoms are $1.0 \mathrm{eV}$ and $0.1 \mathrm{eV}$, respectively, toward each $\mathrm{OH}$ stretch. The kinetic energy difference between $\mathrm{H}^{2}$ and $\mathrm{H}^{3}$ reflects that of the $\mathrm{OH}$ bond strengths. Because the water molecule including the $\mathrm{H}^{3}$ is bonded to the $\mathrm{Mn}$ atom, the $\mathrm{O}-\mathrm{H}^{3}$ bond can easily dissociate if the $\mathrm{Mn}$ is oxidized. The initial electronwavepacket is prepared as a coherent superposition of the photoexcited states. We actually superpose the excited-states weighted 

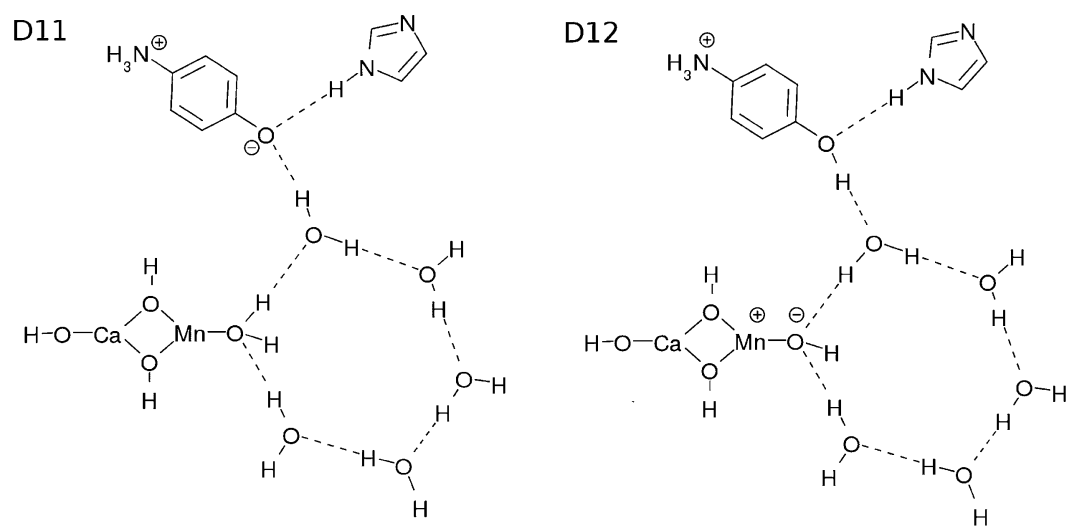

D13
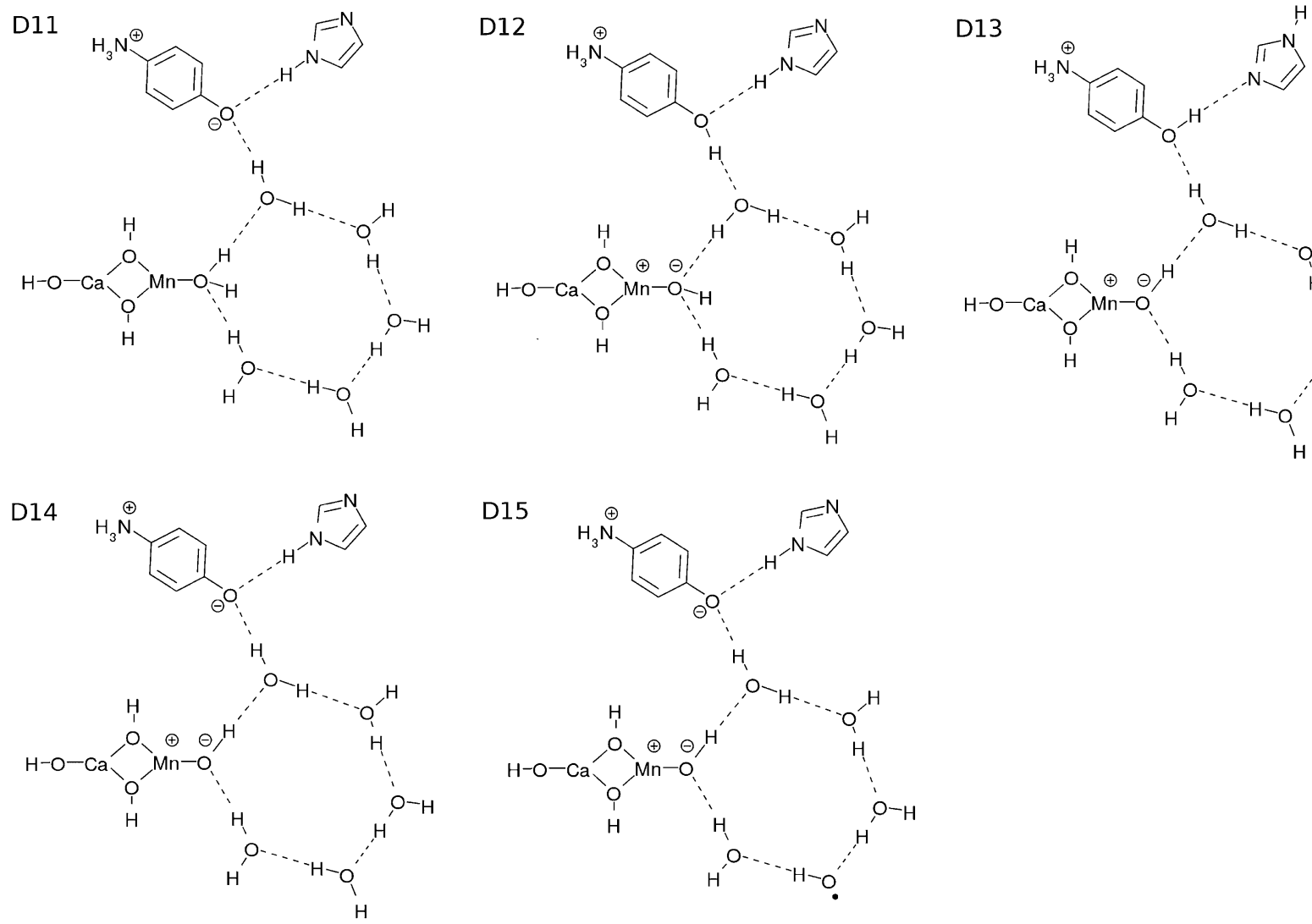

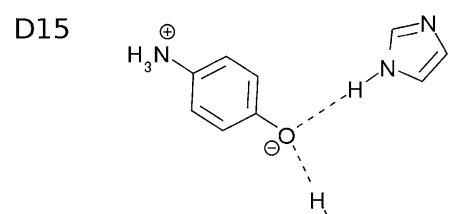

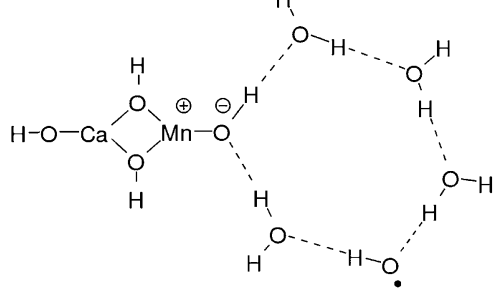

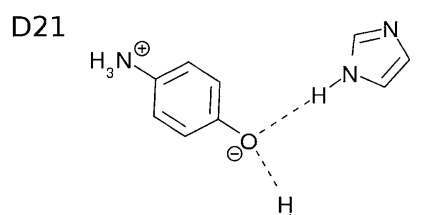

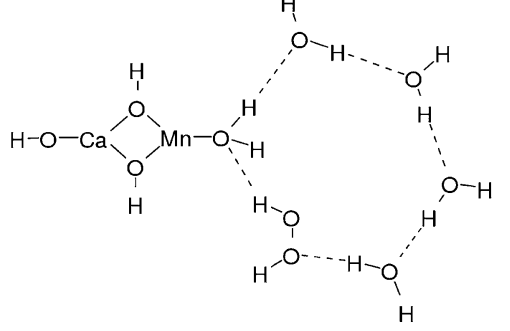

D22<smiles>[O]c1ccc(OCCn2ccnc2)cc1</smiles>

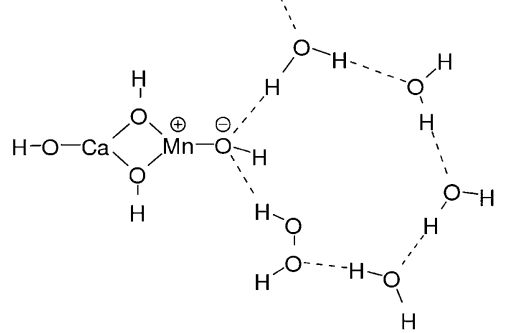

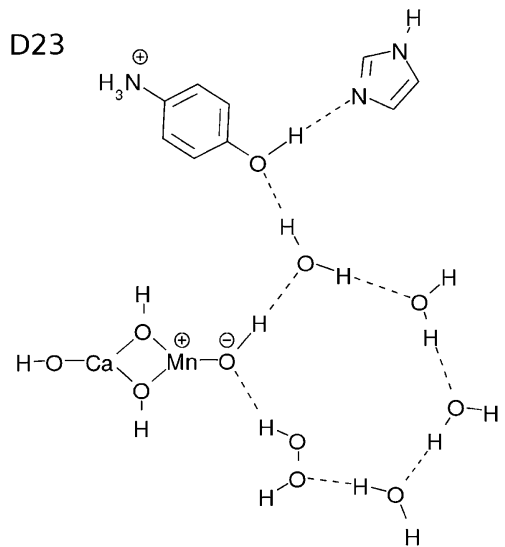

D24<smiles></smiles>

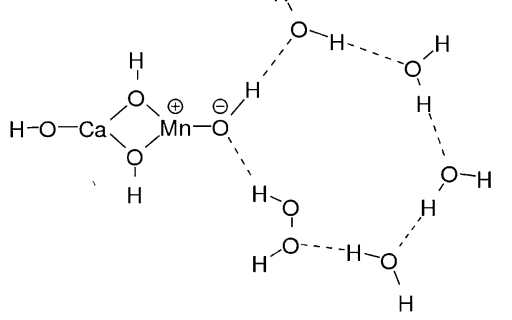

D25

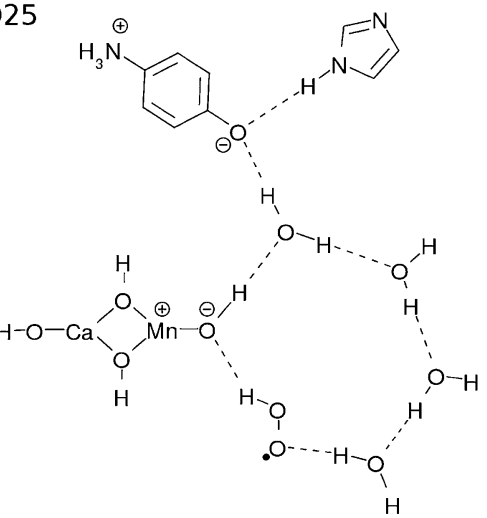

Fig. 8 Projection of the local minima of the key structures. 
a) D11

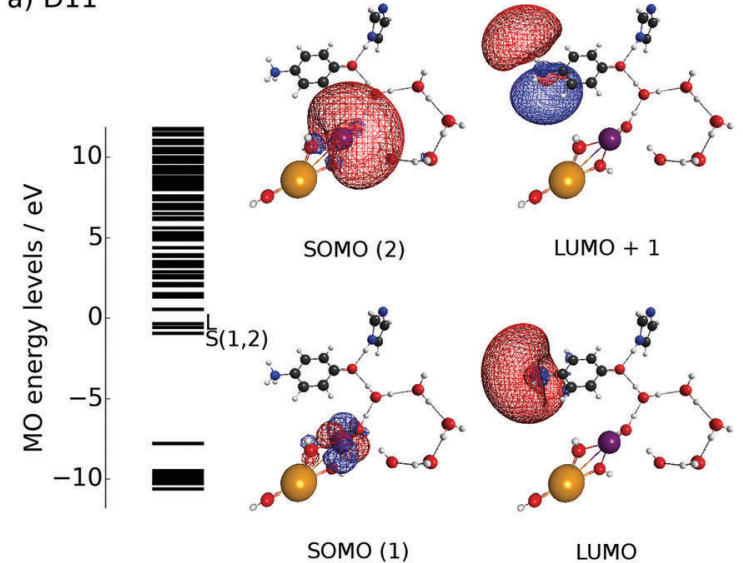

b) D21

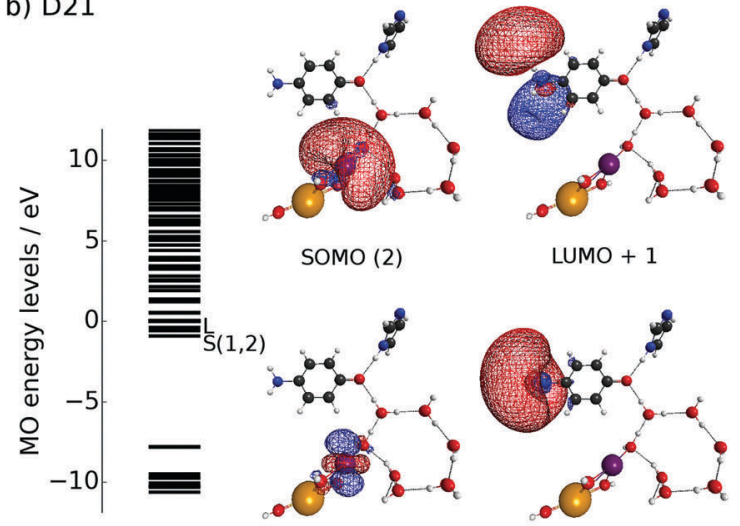

SOMO (1)

LUMO

Fig. 9 Spatial distribution of the frontier MOs of the initial geometry for (a) D11 and (b) D21. Each geometry can be regarded as a subsystem of $K_{n}$, in which $n=0$ or 1 for D11 and $n=2$ or 3 for D21.

with the oscillator strength (see Section S1, ESI $\dagger$ ). The photoexcited states have an energy gap of around $3 \mathrm{eV}$ from the ground state. The initial mean potential energy is approximately equal to $3 \mathrm{eV}$ in both cases of D11 and D21. We have practically tried a number of choices of nuclear momentum and confirmed that the phenomena we have observed are qualitatively robust. Here we pick from among them a typical path for clear illustration.

3.3.3 Photoexcited-state proton and electron dynamics. The resulting dynamics thus observed turned out to be indeed similar to those of the previous study without the hydrogenbond network between the EPD and the EA, ${ }^{5}$ in which the protons and electrons took mutually different pathways to reach spatially different places to result in charge separation. This numerically observed fact is critically important in the study of photoinduced charge separation dynamics through hydrogen-bonding networks and/or also in the spin triplet states. It turns out that CPEWT is thus universal to this extent.

Because the results for D11 and D21 are qualitatively the same as each other, we will first explain the results for D11, and briefly discuss D21 later. Let us track the electron dynamics associated with the $\mathrm{H}^{2}$ and $\mathrm{H}^{3}$ relay-transfer. Recall that the $\mathrm{H}^{1}$ transfer had already occurred in the ground state, which is regarded as one of the proton transfers of the relay. In Fig. 10a, we show selected snapshots of the spatial distribution of the

a) D11
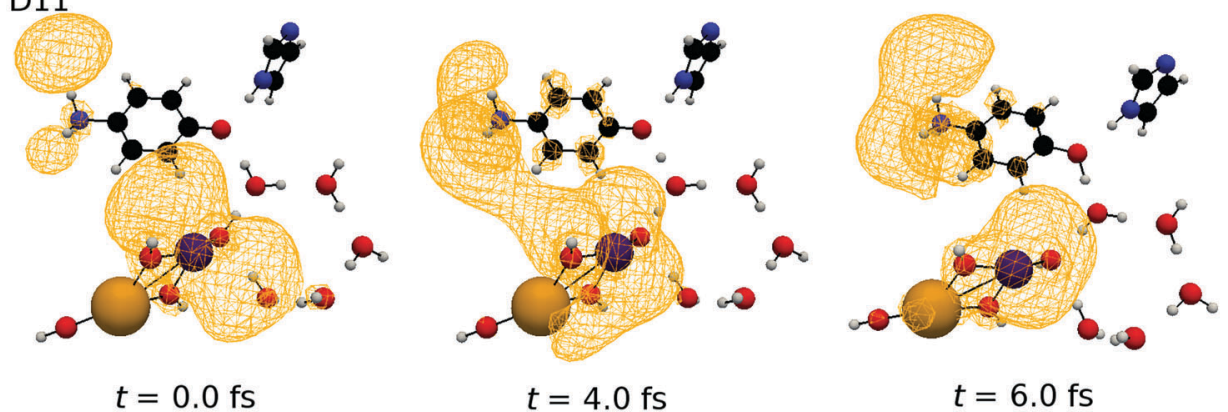

$t=4.0 \mathrm{fs}$

$t=6.0 \mathrm{fs}$

b) D21
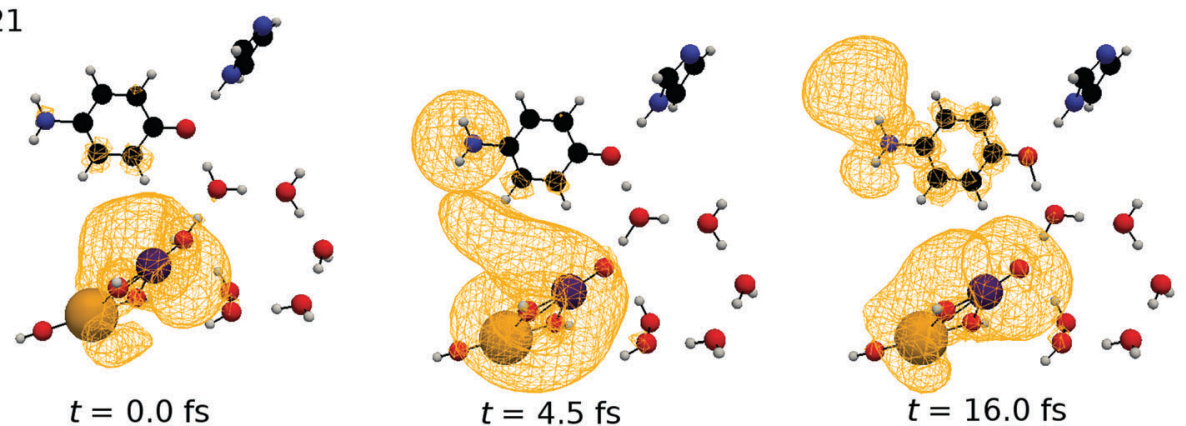

Fig. 10 Selected snapshots of the spatial distribution of the unpaired electron density $D(\mathbf{r})$ (yellow contour mesh) along the photoexcited SET paths of D11 (a) and D21 (b). Color is assigned as follows: $\mathrm{Mn}=$ purple, $\mathrm{Ca}=$ orange, $\mathrm{O}=$ red, $\mathrm{N}=$ blue, $\mathrm{C}=$ black and $\mathrm{H}=$ gray. 
unpaired electron density $D(\mathbf{r})$ for D11, which is defined as follows: ${ }^{67}$

$$
D(\mathbf{r})=2 \rho(\mathbf{r}, \mathbf{r})-\int \mathrm{d} \mathbf{r}^{\prime} \rho\left(\mathbf{r}, \mathbf{r}^{\prime}\right) \rho\left(\mathbf{r}^{\prime}, \mathbf{r}\right)
$$

in which $\rho\left(\mathbf{r}, \mathbf{r}^{\prime}\right)$ is the first order spinless density matrix in the coordinate representation. At $t=0.0 \mathrm{fs}$, right after the photoexcitation and before the $\mathrm{H}^{2}-\mathrm{H}^{3}$ proton relay-transfer, the majority of the unpaired electrons stay on the EPD as a localized biradical pair. And then some part is transferred to the Rydberg-like states of the EA to bring about the asymptotic (spatially well-separated) biradical state. The unpaired electrons take different pathways from those of both $\mathrm{H}^{2}$ and $\mathrm{H}^{3}$. The proton relay-transfer is seen as sequential linear motions, while the motion of the unpaired electrons to reach the Rydberg-like states looks rather circular. This is one of the general features of CPEWT, which has been identified many times in our previous studies. ${ }^{3-5}$ The snapshots for D21, carrying essentially the same information as those of D11, are shown in Fig. 10b. Note that the $\mathrm{H}_{2} \mathrm{O}_{2}$ in the water cluster has virtually no contribution to the CPWET. See Section S1 (ESI $\dagger$ ) for the regional population analysis to further characterize the CPEWT of the present system.

\subsection{Energy profile for the series of reactions}

We next survey the feasibility of the chemical reactions involved in the catalytic cycle from the view point of the energetics only. The computations of relevant chemical dynamics are prohibitively large in the present stage of our study, and it is also very difficult to faithfully track the reaction coordinates in so many dimensional complicated reactions. For the same reason, studies to identify mechanisms such as the coherent dynamics, concerted reactions, or thermal processes were not performed. Instead we only picked up key molecular configurations and examined their energy profile in terms of the standard quantum chemistry.

3.4.1 Method of energy calculation. To estimate the energy profile of the proposed K-state cycle, we divided the system into two groups. The first one contained the EA, PA, EPD, EPR, and $\mathrm{H}^{n}$ (see Fig. 2 to identify the symbols), and the second one consisted of all of the other parts including the XEA, XPA, EBf, and PBf (see Fig. 8). As for the first group, we explicitly regarded them as super-molecules and optimized the geometry for each intermediate. For the second group, we only took account of the reaction energies, which will be described below. The gross reaction energy of the entire system was given as the sum of these two groups.

When the system starts from the configuration of $\mathrm{K}_{0}$, its first group is ${ }^{3}$ [D11] (D11 in structure and triplet in spin). The second group consisted of EBf- $\mathrm{e}^{-}, \mathrm{PBf}-\mathrm{H}^{+}, 4 \mathrm{XEA}, 4 \mathrm{XPA}$, and $2 \mathrm{H}_{2} \mathrm{O}$. In other words, the second group had two water molecules, the buffers with an electron and a proton, and the empty external proton and electron acceptors (see Fig. 2). After the CPEWT and the electron abstraction from the Rydberg-like states of the EA, the system changes to $\mathrm{K}_{02}$. The first group becomes ${ }^{2}[\mathrm{D} 12]^{+}$, and one of the XEAs accepts the electron from the first group, that is,
4XEA $+\mathrm{e}^{-} \rightarrow$ 3XEA + XEA- $\mathrm{e}^{-}$. In this way, we can define the system components to draw an energy profile corresponding to the reaction scheme.

Several spin states of product combinations of $K_{35}$ ([D11]) and oxygen molecule are possible quantum mechanically in the final stage of the series of reactions, even though the initial spin state of $K_{0}$ is triplet. For instance, the spin combinations ${ }^{3}[\mathrm{D} 11]+{ }^{3} \mathrm{O}_{2},{ }^{1}[\mathrm{D} 11]+{ }^{3} \mathrm{O}_{2},{ }^{3}[\mathrm{D} 11]+{ }^{1} \mathrm{O}_{2}$ are allowed starting from ${ }^{3} \mathrm{~K}_{0}$, depending on the energy available, where ${ }^{3}[\mathrm{D} 11]$ indicates the triplet of D11. Obviously, ${ }^{3}[\mathrm{D} 11]+{ }^{3} \mathrm{O}_{2}$ is the lowest in energy and brings the system back to ${ }^{3} K_{0}$. However, due to the limitation of the computational capacity available, we actually computed a little higher energy channel to ${ }^{1}[\mathrm{D} 11]+{ }^{3} \mathrm{O}_{2}$. We thus defined the $\mathrm{K}_{0}{ }^{\prime}$ state, which is a spin singlet state corresponding to the triplet $\mathrm{K}_{0}$. It includes ${ }^{1}[\mathrm{D} 11]$ and ${ }^{3} \mathrm{O}_{2}$. See Section S2 (ESI $\dagger$ ) for further details of the energy profile estimation.

We had ten types of the first group labeled Dnm $(n=1$ or 2 and $m=1,2,3,4$ or 5), as shown in Fig. 8. D1 $m$ appears in $K_{0}-K_{1}$ and $\mathrm{K}_{1}-\mathrm{K}_{2}$ transitions, whereas $\mathrm{D} 2 \mathrm{~m}$ appears in $\mathrm{K}_{2}-\mathrm{K}_{3}$ and $\mathrm{K}_{3}-\mathrm{K}_{0}$ transitions. The major difference between $\mathrm{D} 1 \mathrm{~m}$ and D2 $m$ lies in that $\mathrm{H}_{2} \mathrm{O}_{2}$ is included in the EPR in D2 $m$ but not in that of $\mathrm{D} 1 \mathrm{~m}$. We estimated the energy of the first group for Dnm with the open-shell RHF level of calculation after geometry optimization except for ${ }^{1}$ [D11].

As for the second group, its constituent molecules have not yet been explicitly specified. This is because we have thus far discussed only the functionality of components like EBf and PBf acting in the photocatalytic cycle. To examine the energetics of the second group, we here dare to specify possible molecular species as appropriate candidates for the EBf and PBf. It seems natural to consider the energy profiles of redox reactions of 4-methylphenol as a model of tyrosine since it is used to store and carry electrons and protons in biochemical systems, ${ }^{39-41}$ suggesting that the energy range involved in the water-splitting cycle should also be suitable for the present model system. Thus we estimated the energy difference between XEA and XEA- $\mathrm{e}^{-}$from the following simple case, namely, the reaction of the 4-methylphenyl radical and 4-methylphenyl anion:

$$
\mathrm{CH}_{3}-\mathrm{C}_{6} \mathrm{H}_{4}-\mathrm{O}+\mathrm{e}^{-} \rightarrow\left[\mathrm{CH}_{3}-\mathrm{C}_{6} \mathrm{H}_{4}-\mathrm{O}\right]^{-}
$$

The energy difference between EBf and EBf- $\mathrm{e}^{-}$was calculated in a similar way to those for XEA and EXA- $\mathrm{e}^{-}$for simplicity. Similarly, we estimated the energy difference between XPA and $\mathrm{XPA}-\mathrm{H}^{+}$from the reaction of 4-methylphenyl anion and 4-methylphenol:

$$
\left[\mathrm{CH}_{3}-\mathrm{C}_{6} \mathrm{H}_{4}-\mathrm{O}\right]^{-}+\mathrm{H}^{+} \rightarrow \mathrm{CH}_{3}-\mathrm{C}_{6} \mathrm{H}_{4}-\mathrm{OH}
$$

The energy difference between $\mathrm{PBf}$ and $\mathrm{PBf}-\mathrm{H}^{+}$was calculated in the same way as for XPA and XPA- $\mathrm{H}^{+}$.

For the proton and/or electron exchange between the first and second groups, we only considered the energy changes to assess whether such a reaction is energetically possible or not, and we did not explicitly consider the mechanism of proton and/or electron exchange between the first and second groups. Incidentally, we have studied the reaction between the 3-methylindolyl radical and 3-methylindole as a model of 
tryptophan as another candidate of the above redox reactions and found that a wider energy range is needed to materialize water-splitting in the present cycle.

3.4.2 Global energy profile. The resulting energy profile corresponding to the present water-splitting cycle is shown in Fig. 11. The horizontal axis designates the intermediate $\mathbf{K}_{m n}$. $K_{16}$ and $K_{36}$ are omitted in Fig. 11 to save space, since they have as much energy as $\mathrm{K}_{2}$ and $\mathrm{K}_{0}{ }^{\prime}$, respectively. The states involved in the $K_{n}-K_{n 1}$ transition ( $n=0,1,2$ or 3 ) corresponding to the CPEWT are expressed so as to have some energy range to reflect the quasi-degenerate states in the excited states. See Section S2 of the ESI $\dagger$ for details of the energy profile.

The energy profile can be divided into four parts, in which each corresponds to the $\mathrm{K}_{n}-\mathrm{K}_{n+1}$ transition and starts with photoexcitation followed by CPEWT. The final reaction is $\mathrm{O}=\mathrm{O}$ bond formation, the mechanism of which will be further studied below. The energy of the resulting system $\mathrm{K}_{0}{ }^{\prime}$ is not equal to that of $\mathrm{K}_{0}$. The difference originates from the spin multiplicity; $\mathrm{K}_{0}$ is in the spin triplet state and ends up in singlet state as $\mathrm{K}_{0}{ }^{\prime}$ after releasing a triplet oxygen molecule. It can be seen that the stable intermediates expressed as black bars in this figure lie in the range of $-2 \mathrm{eV}$ to $+3 \mathrm{eV}$. We did not find a state with extremely high or low energy. As far as the photon energy $(\sim 3 \mathrm{eV})$ and energies of the metastable intermediates are concerned, we may say that this water-splitting cycle is possible.

\section{5 $\mathrm{O}=\mathrm{O}$ bond formation to reduce the Mn oxides}

Now let us discuss the mechanism of triplet $\mathrm{O}_{2}$ generation via the formation of $\mathrm{H}_{2} \mathrm{O}_{2}$. As will be demonstrated below, the

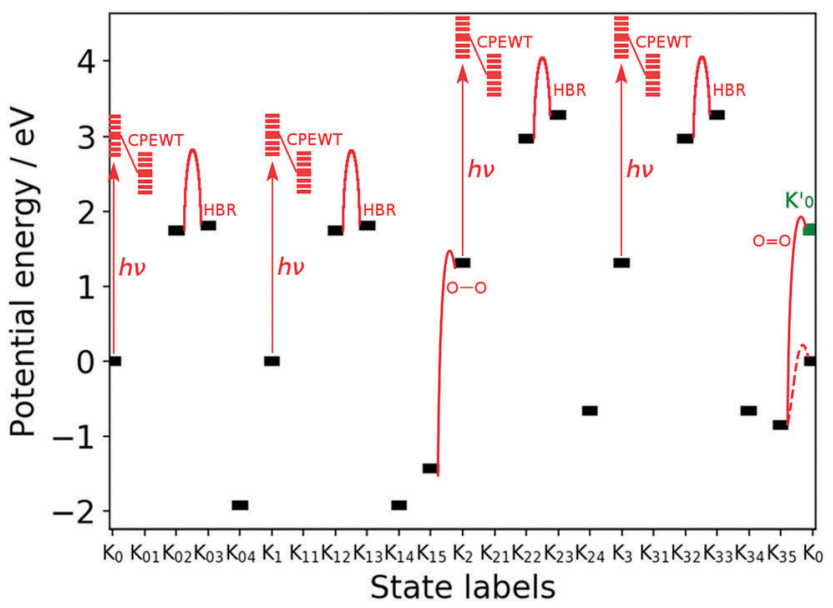

Fig. 11 Energy of the key stages designated by $\mathrm{K}_{n m}$ of the reaction

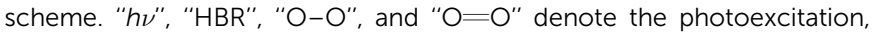
hydrogen-bond reorganization, and $\mathrm{O}-\mathrm{O}$ bond formation as $\mathrm{H}_{2} \mathrm{O}_{2}$, and the $\mathrm{O}=\mathrm{O}$ bond formation as $\mathrm{O}_{2}$, respectively. The barrier heights of the HBR are estimated to be at most $1.22 \mathrm{eV}$ for $\mathrm{K}_{02} \rightarrow \mathrm{K}_{03}$ and $1.16 \mathrm{eV}$ for $\mathrm{K}_{22} \rightarrow \mathrm{K}_{23}$. Those for $\mathrm{K}_{12} \rightarrow \mathrm{K}_{13}$ and $\mathrm{K}_{32} \rightarrow \mathrm{K}_{33}$ are the same as those for $\mathrm{K}_{02} \rightarrow \mathrm{K}_{03}$ and $\mathrm{K}_{22} \rightarrow \mathrm{K}_{23}$, respectively. The energy of $\mathrm{K}_{n m}$ is that before the next process begins on it. For instance the energy of $\mathrm{K}_{04}$ (see Fig. 4) does not contain the energy required for protonation and electron-attachment to take place on it next. See text for the definition of $K_{0}{ }^{\prime}$ that is colored in green. The dashed line connecting $K_{35}$ and $K_{0}$ is a possible pathway without singlet intermediates, not going to $\mathrm{K}_{0}{ }^{\prime}$. mechanism of the $\mathrm{O}=\mathrm{O}$ (as in $\mathrm{O}_{2}$ ) and $\mathrm{O}-\mathrm{O}$ (as in $\mathrm{H}_{2} \mathrm{O}_{2}$ ) bond formations are similar to each other. First we recall that both the reactant and product have been shown in the $\mathrm{K}_{n 5}-\mathrm{K}_{n 6}$ transitions in Section 2, in which $n=1$ and $n=3$ for $\mathrm{O}-\mathrm{O}$ (Fig. 5) and $\mathrm{O}=\mathrm{O}$ (Fig. 7), respectively. Here we seek a deeper insight into the mechanism through a qualitative analysis of the electronic states. The present model asserts that they are both born from the water cluster and are associated with the reduction of the $\mathrm{Mn}$ oxide in such a way to make the next CPEWT possible. Since it is very difficult and time-consuming to carry out dynamical calculations, we instead tracked the evolution of the ground-state properties along an appropriate proton-transfer coordinate leading to $\mathrm{O}-\mathrm{O} / \mathrm{O}=\mathrm{O}$ bond formations.

We employed the CISD/RHF (triplet) level of calculation for the electrically neutral subsystem consisting of the EPD and a part of the EPR that is necessary for $\mathrm{O}-\mathrm{O} / \mathrm{O}=\mathrm{O}$ bond formation. The CISD active space was chosen as follows: (HOMO-9)-HOMO and two SOMOs were set to be the active occupied MOs, whereas the LUMO was the active virtual MO. This active space including the $\mathrm{p}$ and $\mathrm{d}$ orbitals of the $\mathrm{O}$ and the $\mathrm{Mn}$ atoms, respectively, can qualitatively describe the change of electronic states enough to clarify the mechanism of the $\mathrm{O}-\mathrm{O} / \mathrm{O}=\mathrm{O}$ bond formations.

3.5.1 Proton transfer followed by $\mathrm{O}-\mathrm{O} / \mathrm{O}=\mathrm{O}$ bond formation. We illustrate the mechanism of triplet $\mathrm{O}_{2}$ generation by using a set of constraint-optimized geometries of the subsystem. Because of the similarity of the bond formation mechanism of $\mathrm{O}-\mathrm{O}$ and $\mathrm{O}=\mathrm{O}$, we first discuss the $\mathrm{O}-\mathrm{O}$ bond formation and will mention the mutual differences later. A typical case of proton transfer to the EPD is discussed first. Other possible mechanisms have been examined, including simultaneous proton transfer and $\mathrm{O}-\mathrm{O}$ bond formation, and these reaction pathways turned out to need significantly higher energy barriers than the present mechanism.

We concentrated on the four atoms of the subsystem to consider the mechanism. They are indicated by blue circles in Fig. 12a. The leftmost configuration is an excerpt of the essential subsystem of $K_{15}$ (see Fig. 5). The subsystem itself was optimized beforehand, and no significant difference from the original one in $\mathrm{K}_{15}$ was found. As for the first reaction (proton transfer), the $\mathrm{H}$ atom in $\mathrm{H}_{2} \mathrm{O}$ that is doubly circled in the leftmost panel is forced to almost linearly shift to the EPD site so that the $\mathrm{OH}$ bond length is shortened down to $1.0 \AA$, which is the typical $\mathrm{OH}$ bond length. The other three circled atoms are fixed in space while this reaction, and the other atoms are optimized. This is therefore a constrained geometry optimization. By this proton shift, the molecular configuration becomes as shown in the central panel of Fig. 12a. The spatial distribution of the unpaired electron density does not significantly change in this reaction, that is, the subsystem remains in a biradical state.

Next the $\mathrm{O}$ atom of the $\mathrm{OH}$ radical that is doubly circled in the central panel is shifted to the $\mathrm{O}$ atom of the other $\mathrm{OH}$ that has been created by the first proton transfer. Hence, the $\mathrm{OO}$ bond length is shortened to $1.5 \AA$, which is the typical O-O bond length of $\mathrm{H}_{2} \mathrm{O}_{2}$. This approach of two oxygen atoms results in the geometry in the rightmost panel of Fig. 12a. The exceeding electron for $\mathrm{H}_{2} \mathrm{O}_{2}$ is transferred to the EPD, 
a) $\mathrm{H}_{2} \mathrm{O}_{2}$ generation

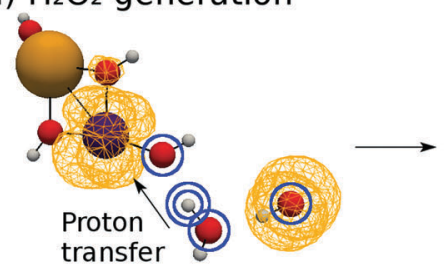

$(0 \mathrm{eV}, 0 \mathrm{eV})$
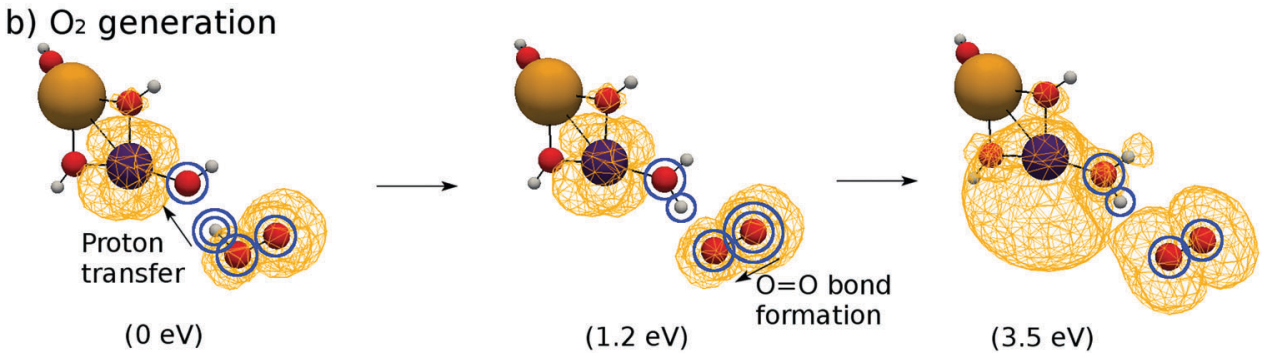

$(3.5 \mathrm{eV})$

Fig. 12 Spatial distribution of the unpaired electron density relevant to the bond formation of (a) $\mathrm{H}_{2} \mathrm{O}_{2}$ and (b) $\mathrm{O}_{2}$. The leftmost figures are optimized normally, and the center and rightmost figures depict the constraint-optimized geometry. The values in the parentheses in black beneath each snapshot denote the energy difference from the initial state, whereas the values in red show similar quantities except that the system is surrounded by 20 water molecules as a solvent. oxidizing the EPR, which is seen as the unpaired electron density localizing on the EPD as shown in the rightmost panel of Fig. 12a.

The energy increases rather monotonically from the left configuration to the right one in the panel of Fig. 12a, the barrier of which amounts up to about $3.9 \mathrm{eV}$. This energy difference is higher than the energy profile shown in Fig. 11, which is equal to $3.0 \mathrm{eV}$. This difference mainly comes from the way of calculation in Fig. 12a, which represents the calculation under constrained geometry optimization with naked (gas phase) reaction disregarding the presence of the solvent water molecules (see the electronic energy for each configuration in the parentheses in Fig. 12). In fact, the generated HOOH should actually be stabilized by hydrogen bonds as shown in $\mathrm{K}_{2}$ of Fig. 5. It is therefore natural to conceive that this series of reactions takes place cooperatively in the solvation dynamics being surrounded by abundant water molecules. Indeed, if we add many more water molecules (actually about 20 molecules) such that three water molecules reside surrounding the relevant $\mathrm{H}_{2} \mathrm{O}_{2}$, the energy barrier lowers to $2.4 \mathrm{eV}$ with no change of the terminal electronic states (see the values in red in the parentheses in Fig. 12a). Therefore, this reaction can take place within the energy range of the $\pm 3 \mathrm{eV}$.

The pathway of $\mathrm{O}=\mathrm{O}$ bond formation is implemented similarly to that of $\mathrm{O}-\mathrm{O}$ bond formation (see Fig. 12b), but the minimum OO bond length is employed as $1.2 \AA$, which is the typical length for molecular oxygen in the triplet state. The energy barrier for the subsystem is about $3.6 \mathrm{eV}$, which is slightly less than that of the $\mathrm{O}-\mathrm{O}$ bond formation. The proton and electron transfers occur in the same manner to reduce the EPD, but their spin distributions are totally different from each other as shown in the rightmost panels of Fig. 12a and b, which is discussed below.
3.5.2 The spin multiplicity between the $\mathrm{O}-\mathrm{O}$ and $\mathrm{O}=\mathrm{O}$ bond formations. The $\mathrm{O}-\mathrm{O}$ and $\mathrm{O}=\mathrm{O}$ bond formations are quite similar to each other except in one important aspect of spin multiplicity. As seen in Fig. 12, both reactions begin from biradical states, in which the unpaired electrons are located mainly on both the EPD and EPR sites. However, in the O-O bond formation the unpaired electrons are transferred to the EPD site to restore the localized triplet biradical state (see the rightmost panel of Fig. 12). The number of unpaired electrons is about 2.0. On the other hand, the $\mathrm{O}=\mathrm{O}$ bond formation ends up with its unpaired electron distribution found on both the EPD and the EPR sides (see the rightmost panel of Fig. 12b). The number of unpaired electrons is thus about $4.0(2.0$ on each). Analysis on the spin distribution shows that this tetra-radical state represents triplet $\mathrm{O}_{2}$ and the triplet (or even the singlet) biradical EPD simultaneously. This is one of the pathways of triplet $\mathrm{O}_{2}$ generation from singlet water molecules.

\section{Concluding remarks}

In terms of the dynamics and energetics of small Mn-oxides surrounded and contacted by hydrogen-bonding networks of water molecules, we have proposed a novel mechanism of the water-splitting photocatalytic cycle triggered by coupled proton electron-wavepacket transfer (CPEWT) to induce charge separation and have shown that the system is indeed feasible from the view point of energetics. This mechanism is a four-photon process from which a triplet $\mathrm{O}_{2}$ molecule emerges via the temporal formation of $\mathrm{HOOH}$ in the water cluster. We theoretically constructed a reaction scheme of the water-splitting cycle based on the characteristics of the CPEWT and the theoretically 
estimated energy profile, thereby showing that all of the metastable intermediates lie in the energy range of $\pm 2.5 \mathrm{eV}$. Since each photon absorbed in the process of CPEWT has an energy of approximately $3 \mathrm{eV}$, the water-splitting cycle can be judged to be feasible.

In the present scheme the Mn-oxides are supposed to be directly photoexcited up to an electronic manifold in which proton transfer causes nonadiabatic electron wavepacket transfer through pathways different from each other, thereby resulting in charge separation in the acceptors. ${ }^{3-5}$ This physical assumption may not be applied to the dynamics in PSII, which is widely conceived to take place in the electronic ground state. ${ }^{1,2}$ Therefore the present study is concerned with the very basic mechanism of photocatalysts and may be useful for designing artificial water splitting and/or solar cells. In this study of photodynamics, it has been positively confirmed that the CPEWT dynamics can take place even in the presence of hydrogen-bonding networks and also in the spin triplet state. As a result, it has been shown that triplet $\mathrm{O}_{2}$ can be generated from water clusters via $\mathrm{H}_{2} \mathrm{O}_{2}$ as an intermediate. It turns out that none of the oxygen atoms of the catalytic center $\mathrm{MnCaO}_{4} \mathrm{H}_{5}$ are directly involved in the $\mathrm{O}=\mathrm{O}$ bond formation.

Before closing this paper, let us briefly mention the implication of the difference between our proposed mechanism of $\mathrm{O}_{2}$ generation and that proposed by Siegbahn $^{68}$ and other experimentalists ${ }^{69,70}$ studying $\mathrm{Mn}_{4} \mathrm{CaO}_{5}$ involved in PSII. The latter is summarized in that $\mathrm{O}_{2}$ is formed by breaking one of the $\mu$-oxo bridges of the Mn cluster without tentative generation of peroxide $(\mathrm{HOOH})$ (as for the possible formation of peroxides in the moiety of PSII, see ref. 71-74.) Besides, it is widely believed that virtually all plants and cyanobacteria carrying out photosynthesis resort exclusively to $\mathrm{Mn}_{4} \mathrm{CaO}_{5}$ as the central catalyst. ${ }^{75}$ These studies on $\mathrm{Mn}_{4} \mathrm{CaO}_{5}$ raise a naive question about evolution at the molecular level, since it is hard to imagine that such a highly sophisticated cluster and mechanism appeared all of sudden in the evolutionary process of biological systems (allegedly 2.5 billion years ago ${ }^{76,77}$ ). It is quite likely that simpler and less sophisticated photocatalysts were used by biological systems on the way to the goal of the current biological status resting on $\mathrm{Mn}_{4} \mathrm{CaO}_{5}$. Not only the evolution of catalysts but the composition of the air on the earth should be different from what we have now in that the amount of oxygen should have been far less and consequently the distribution of the wavelength of the sunlight to the earth surface must have been shifted to the ultraviolet region. All of these factors suggest that photocatalytic cycles in the wilder and immature circumstances of mother nature should have used smaller Mn oxides and simpler mechanisms for charge separation and giving birth to oxygen molecules directly from bulk water before the biological systems finally attained $\mathrm{Mn}_{4} \mathrm{CaO}_{5}$.

From the view point of artificial water splitting technology, an appropriate choice of electron buffer and proton buffer can be crucial to determine the efficiency of splitting. In this paper we tentatively assigned the 4-methylphenyl radical as a candidate of the electron buffer, and the 4-methylphenyl anion as that of the proton buffer. In this regard, another comparison between our proposed photocatalytic system basically with $\mathrm{MnCaO}_{4} \mathrm{H}_{5}$ and $\mathrm{Mn}_{4} \mathrm{CaO}_{5}$ in PSII suggests that the roles of electron buffer necessary for the regulation of charge and spin multiplicity are achieved in a sophisticated manner by the presence of multiple $\mathrm{Mn}$ atoms in $\mathrm{Mn}_{4} \mathrm{CaO}_{5}$. This aspect will be discussed in the future.

Finally, one of the most difficult aspects in charge separation dynamics in water splitting by PSII is the widely accepted assumption that it can take place in the electronic ground state. $^{1,2}$ This is not a matter of biological assumption but is about chemical principle. In our next paper we will discuss the possibility of ground-state CPEWT in Mn oxides and accepters, a charge separation which is induced by collision with an external chromophore.

\section{Conflicts of interest}

There are no conflicts of interest to declare.

\section{Acknowledgements}

This work was supported in part by a Grant-in-Aid for Scientific Research from the Ministry of Education and Science in Japan (15H05752).

\section{References}

1 D. Voet and J. G. Voet, Biochemistry, John Wiley, New Jersey, 4th edn, 2011.

2 J. M. Berg, J. L. Tymoczko and L. Stryer, Biochemistry, Freeman, New York, 5th edn, 2002.

3 K. Yamamoto and K. Takatsuka, ChemPhysChem, 2015, 16, 2534-2537.

4 K. Yamamoto and K. Takatsuka, Chem. Phys., 2016, 475, 39-53.

5 K. Yamamoto and K. Takatsuka, ChemPhysChem, 2017, 18, 537-548.

6 H. Ushiyama and K. Takatsuka, Angew. Chem., 2007, 119, 593-596.

7 S. Hammes-Schiffer, Chem. Rev., 2010, 110, 6937-6938.

8 S. Hammes-Schiffer and A. A. Stuchebrukhov, Chem. Rev., 2010, 110, 6939-6960.

9 M. H. V. Huynh and T. J. Meyer, Chem. Rev., 2007, 107, 5004-5064.

10 O. Tishchenko, D. G. Truhlar, A. Ceulemans and M. T. Nguyen, J. Am. Chem. Soc., 2008, 130, 7000-7010.

11 S. Hammes-Schiffer and A. V. Soudackov, J. Phys. Chem. B, 2008, 112, 14108-14123.

12 P. E. Siegbahn and M. R. Blomberg, Chem. Rev., 2010, 110, 7040-7061.

13 S. Hammes-Schiffer, J. Phys. Chem. Lett., 2011, 2, 1410-1416.

14 D. R. Weinberg, C. J. Gagliardi, J. F. Hull, C. F. Murphy, C. A. Kent, B. C. Westlake, A. Paul, D. H. Ess, D. G. McCafferty and T. J. Meyer, Chem. Rev., 2012, 112, 4016-4093. 
15 J. P. Layfield and S. Hammes-Schiffer, Chem. Rev., 2014, 114, 3466-3494.

16 A. L. Sobolewski and W. Domcke, J. Phys. Chem. A, 2001, 105, 9275-9283.

17 A. Sobolewski, W. Domcke, C. Dedonder-Lardeux and C. Jouvet, Phys. Chem. Chem. Phys., 2002, 4, 1093-1100.

18 A. L. Sobolewski and W. Domcke, J. Phys. Chem. A, 2007, 111, 11725-11735.

19 A. L. Sobolewski and W. Domcke, J. Phys. Chem. A, 2008, 112, 7311-7313.

20 A. Carrera, I. Nielsen, P. Carcabal, C. Dedonder, M. Broquier, C. Jouvet, W. Domcke and A. Sobolewski, J. Chem. Phys., 2009, 130, 024302.

21 R. Borrelli and W. Domcke, Chem. Phys. Lett., 2010, 498, 230-234.

22 R. Borrelli, M. Thoss, H. Wang and W. Domcke, Mol. Phys., 2012, 110, 751-763.

23 A. L. Sobolewski and W. Domcke, Chem. Phys. Lett., 2009, 479, 144-148.

24 A. L. Sobolewski and W. Domcke, Phys. Chem. Chem. Phys., 2012, 14, 12807-12817.

25 T. N. Karsili, D. Tuna, J. Ehrmaier and W. Domcke, Phys. Chem. Chem. Phys., 2015, 17, 32183-32193.

26 X. Liu, A. L. Sobolewski, R. Borrelli and W. Domcke, Phys. Chem. Chem. Phys., 2013, 15, 5957-5966.

27 X. Liu, A. L. Sobolewski and W. Domcke, J. Phys. Chem. A, 2014, 118, 7788-7795.

28 X. Liu, T. N. Karsili, A. L. Sobolewski and W. Domcke, J. Phys. Chem. B, 2015, 119, 10664-10672.

29 X. Liu, T. N. Karsili, A. L. Sobolewski and W. Domcke, Chem. Phys., 2016, 464, 78-85.

30 J. Ehrmaier, T. N. Karsili, A. L. Sobolewski and W. Domcke, J. Phys. Chem. A, 2017, 121, 4754-4764.

31 J. Ehrmaier, D. Picconi, T. N. Karsili and W. Domcke, J. Chem. Phys., 2017, 146, 124304.

32 X. Wu, T. N. Karsili and W. Domcke, Molecules, 2017, 22, 135.

33 W. Domcke, D. Yarkony and H. Köppel, Conical Intersections: Electronic Structure, Dynamics \& Spectroscopy, World Scientific, Singapore, 2004, vol. 15.

34 B. A. Barry, U. Brahmachari and Z. Guo, Acc. Chem. Res., 2017, 50, 1937-1945.

35 B. Kok, B. Forbush and M. McGloin, Photochem. Photobiol., 1970, 11, 457-475.

36 P. Joliot, Photosynth. Res., 2003, 76, 65-72.

37 M.-T. Zhang and L. Hammarström, J. Am. Chem. Soc., 2011, 133(23), 8806-8809.

38 K. Takematsu, H. Williamson, A. M. Blanco-Rodrguez, L. Sokolová, P. Nikolovski, J. T. Kaiser, M. Towrie, I. P. Clark, A. Vlcék Jr and J. R. Winkler, et al., J. Am. Chem. Soc., 2013, 135, 15515-15525.

39 A. Migliore, N. F. Polizzi, M. J. Therien and D. N. Beratan, Chem. Rev., 2014, 114, 3381-3465.

40 H. B. Gray and J. R. Winkler, Proc. Natl. Acad. Sci. U. S. A., 2015, 112, 10920-10925.

41 A. M. Bogdanov, A. Acharya, A. V. Titelmayer, A. V. Mamontova, K. B. Bravaya, A. B. Kolomeisky, K. A. Lukyanov and A. I. Krylov, J. Am. Chem. Soc., 2016, 138, 4807-4817.
42 H.-D. Meyer and W. H. Miller, J. Chem. Phys., 1979, 70, 3214-3223.

43 D. A. Micha, J. Chem. Phys., 1983, 78, 7138-7145.

44 A. Garca-Vela, R. Gerber and D. Imre, J. Chem. Phys., 1992, 97, 7242-7250.

45 W. H. Miller, Faraday Discuss., 1998, 110, 1-21.

46 M. D. Hack, A. W. Jasper, Y. L. Volobuev, D. W. Schwenke and D. G. Truhlar, J. Phys. Chem. A, 2000, 104, 217-232.

47 K. Takatsuka, J. Phys. Chem. A, 2007, 111, 10196-10204.

48 T. Yonehara and K. Takatsuka, J. Chem. Phys., 2008, 129, 134109.

49 T. Yonehara, K. Hanasaki and K. Takatsuka, Chem. Rev., 2012, 112, 499-542.

50 K. Takatsuka, T. Yonehara, K. Hanasaki and Y. Arasaki, Chemical Theory Beyond the Born-Oppenheimer Paradigm: Nonadiabatic Electronic and Nuclear Dynamics in Chemical Reactions, World Scientific, Singapore, 2015.

51 T. Yonehara and K. Takatsuka, J. Chem. Phys., 2008, 128, 154104.

52 T. Yonehara, S. Takahashi and K. Takatsuka, J. Chem. Phys., 2009, 130, 214113.

53 T. Yonehara and K. Takatsuka, Chem. Phys., 2009, 366, 115-128.

54 T. Yonehara and K. Takatsuka, J. Chem. Phys., 2010, 132, 244102.

55 K. Takatsuka and T. Yonehara, Phys. Chem.Chem. Phys., 2011, 13, 4987-5016.

56 K. Takatsuka and T. Yonehara, Adv. Chem. Phys., 2010, 144, 93-156.

57 T. Yonehara and K. Takatsuka, J. Chem. Phys., 2012, 137, 22 A520.

58 T. Yonehara and K. Takatsuka, J. Phys. Chem. A, 2013, 117, 8599-8608.

59 K. Yamamoto and K. Takatsuka, J. Chem. Phys., 2014, 140, 124111.

60 H. Ichikawa and K. Takatsuka, J. Phys. Chem. A, 2016, 121, 315-325.

61 K. Takatsuka, J. Chem. Phys., 2017, 146, 084312.

62 T. Matsuoka and K. Takatsuka, J. Chem. Phys., 2017, 146, 134114.

63 W. J. Stevens, M. Krauss, H. Basch and P. G. Jasien, Can. J. Chem., 1992, 70, 612-630.

64 K. Nagashima and K. Takatsuka, J. Phys. Chem. A, 2012, 116, 11167-11179.

65 M. W. Schmidt, K. K. Baldridge, J. A. Boatz, S. T. Elbert, M. S. Gordon, J. H. Jensen, S. Koseki, N. Matsunaga, K. A. Nguyen, S. Su, T. L. Windus, M. Dupuis and J. A. Montgomery, J. Comput. Chem., 1993, 14, 1347-1363.

66 M. S. Gordon and M. W. Schmidt, Theory and applications of computational chemistry: the first forty years, Elsevier, Amsterdam, 2005.

67 K. Takatsuka, T. Fueno and K. Yamaguchi, Theor. Chim. Acta, 1978, 48, 175-183.

68 P. E. Siegbahn, Acc. Chem. Res., 2009, 42, 1871-1880.

69 M. Suga, F. Akita, M. Sugahara, M. Kubo, Y. Nakajima, T. Nakane, K. Yamashita, Y. Umena, M. Nakabayashi, 
T. Yamane, T. Nakano, M. Suzuki, T. Masuda, S. Inoue, T. Kimura, T. Nomura, S. Yonekura, L.-J. Yu, T. Sakamoto, T. Motomura, J.-H. Chen, Y. Kato, T. Noguchi, K. Tano, Y. Joti, T. Kameshima, T. Hatsui, E. Nango, R. Tanaka, H. Naitow, Y. Matsuura, A. Yamashita, M. Yamamoto, M. Yabashi, T. Ishikawa, S. Iwata and S. Jian-Ren, Nature, 2017, 543, 131-135.

70 I. D. Young, M. Ibrahim, R. Chatterjee, S. Gul, F. D. Fuller, S. Koroidov, A. S. Brewster, R. Tran, R. Alonso-Mori, T. Kroll, T. Michels-Clark, H. Laksmono, R. G. Sierra, C. A. Stan, R. Hussein, M. Zhang, L. Douthit, M. Kubin, C. de Lichtenberg, L. Vo Pham, H. Nilsson, M. H. Cheah, D. Shevela, C. Saracini, M. A. Bean, I. Seuffert, D. Sokaras, T.-C. Weng, E. Pastor, C. Weninger, T. Fransson, L. Lassalle, P. Bräuer, P. Aller, P. T. Docker, B. Andi, A. M. Orville, J. M. Glownia, S. Nelson, M. Sikorski, D. Zhu, M. S. Hunter, T. J. Lane, A. Aquila, J. E. Koglin, J. Robinson, M. Liang, S. Boutet, A. Y. Lyubimov, M. Uervirojnangkoorn, N. W. Moriarty, D. Liebschner, P. V. Afonine, D. G. Waterman, G. Evans, P. Wernet, H. Dobbek, W. I. Weis, A. T. Brunger, P. H. Zwart,
P. D. Adams, A. Zouni, J. Messinger, U. Bergmann, N. K. Sauter, J. Kern, V. K. Yachandra and J. Yano, Nature, 2016, 540, 453-457.

71 L. Rapatskiy, N. Cox, A. Savitsky, W. M. Ames, J. Sander, M. M. Nowaczyk, M. Rögner, A. Boussac, F. Neese, J. Messinger and W. Lubitz, J. Am. Chem. Soc., 2012, 134, 16619-16634.

72 W. Hillier and T. Wydrzynski, Photosynth. Res., 1993, 38, 417-423.

73 P. L. Fine and W. D. Frasch, Biochemistry, 1992, 31, 12204-12210.

74 B. K. Semin, L. N. Davletshina, K. N. Timofeev, I. I. Il'ya, A. B. Rubin and M. Seibert, Photosynth. Res., 2013, 117, 385-399.

75 J. Yano and V. Yachandra, Chem. Rev., 2014, 114, 4175-4205.

76 G. Dismukes, V. Klimov, S. Baranov, Y. N. Kozlov, J. DasGupta and A. Tyryshkin, Proc. Natl. Acad. Sci. U. S. A., 2001, 98, 2170-2175.

77 K. Sauer and V. K. Yachandra, Proc. Natl. Acad. Sci. U. S. A., 2002, 99, 8631-8636. 